\title{
NEW H-BAND STELLAR SPECTRAL LIBRARIES FOR THE SDSS-III/APOGEE SURVEY
}

\author{
O. Zamora ${ }^{1,2}$, D. A. García-Hernández ${ }^{1,2}$, C. Allennde Prieto ${ }^{1,2}$, R. Carrera ${ }^{1,2}$, L. Koesterke $^{3}$, B. Edvardsson ${ }^{4}$, \\ F. Castelli ${ }^{5}$, B. Plez ${ }^{6}$, D. Bizyaev ${ }^{7,8}$, K. Cunha $^{9}$, A. E. García Pérez ${ }^{1,2}$, B. Gustafsson ${ }^{4}$, J. A. Holtzman ${ }^{8}$, J. E. Lawler ${ }^{10}$, \\ S. R. Majewski ${ }^{11}$, A. Manchado ${ }^{1,2,12}$, Sz. Mészáros ${ }^{13}$, N. Shane $^{14}$, M. Shetrone ${ }^{15}$, V. V. Smith ${ }^{16}$, and G. Zasowski ${ }^{17}$ \\ ${ }^{1}$ Instituto de Astrofísica de Canarias, E-38205 La Laguna, Tenerife, Spain; ozamora@iac.es \\ ${ }^{2}$ Departamento de Astrofísica, Universidad de La Laguna (ULL), E-38206 La Laguna, Tenerife, Spain \\ ${ }^{3}$ Texas Advanced Computing Center, The University of Texas at Austin, Austin, TX 78759, USA \\ ${ }^{4}$ Department of Physics and Astronomy, Division of Astronomy and Space Physics, Box 515, SE-751 20 Uppsala, Sweden \\ ${ }^{5}$ Istituto Nazionale di Astrofisica, Osservatorio Astronomico di Trieste, via Tiepolo 11, I-34143 Trieste, Italy \\ ${ }^{6}$ Laboratoire Univers et Particules de Montpellier, Université de Montpellier, CNRS, F-34095 Montpellier, France \\ Apache Point Observatory, P.O. Box 59, Sunspot, NM 88349-0059, USA \\ ${ }_{9}^{8}$ New Mexico State University, Las Cruces, NM 88003, USA \\ ${ }^{9}$ Observatório Nacional, São Cristóvão, Rio de Janeiro, Brazil \\ ${ }^{10}$ Department of Physics, University of Wisconsin-Madison, 1150 University Avenue, Madison, WI 53706, USA \\ ${ }^{11}$ Department of Astronomy, University of Virginia, Charlottesville, VA 22904-4325, USA \\ ${ }^{12}$ Consejo Superior de Investigaciones Cientificas (CSIC) \\ ${ }^{13}$ ELTE Gothard Astrophysical Observatory, H-9704 Szombathely, Szent Imre herceg út, Hungary \\ ${ }^{14}$ Department of Astronomy, University of Virginia, Charlottesville, VA 22904-4325, USA \\ ${ }^{15}$ McDonald Observatory, University of Texas at Austin, Fort Davis, TX 79734, USA \\ ${ }_{16}$ National Optical Astronomy Observatories, Tucson, AZ 85719, USA \\ ${ }^{17}$ Department of Physics and Astronomy, Johns Hopkins University, Baltimore, MD 21218, USA \\ Received 2015 February 18; accepted 2015 March 31; published 2015 May 12
}

\begin{abstract}
The Sloan Digital Sky Survey-III (SDSS-III) Apache Point Observatory Galactic Evolution Experiment (APOGEE) has obtained high-resolution $(R \sim 22,500)$, high signal-to-noise ratio $(>100)$ spectra in the H-band $(\sim 1.5-1.7 \mu \mathrm{m})$ for about 146,000 stars in the Milky Way galaxy. We have computed spectral libraries with effective temperature $\left(T_{\text {eff }}\right)$ ranging from 3500 to $8000 \mathrm{~K}$ for the automated chemical analysis of the survey data. The libraries, used to derive stellar parameters and abundances from the APOGEE spectra in the SDSS-III data release 12 (DR12), are based on ATLAS9 model atmospheres and the ASS $\epsilon$ T spectral synthesis code. We present a second set of libraries based on MARCS model atmospheres and the spectral synthesis code Turbospectrum. The ATLAS9/ASS $\epsilon$ T $\left(T_{\text {eff }}=3500-8000 \mathrm{~K}\right)$ and MARCS/Turbospectrum $\left(T_{\text {eff }}=3500-5500 \mathrm{~K}\right)$ grids cover a wide range of metallicity $(-2.5 \leqslant[\mathrm{M} / \mathrm{H}] \leqslant+0.5 \mathrm{dex})$, surface gravity $(0 \leqslant \log g \leqslant 5 \mathrm{dex})$, microturbulence $(0.5 \leqslant \xi \leqslant$ $\left.8 \mathrm{~km} \mathrm{~s}^{-1}\right)$, carbon $(-1 \leqslant[\mathrm{C} / \mathrm{M}] \leqslant+1 \mathrm{dex})$, nitrogen $(-1 \leqslant[\mathrm{~N} / \mathrm{M}] \leqslant+1 \mathrm{dex})$, and $\alpha$-element $(-1 \leqslant[\alpha / \mathrm{M}] \leqslant+1 \mathrm{dex})$ variations, having thus seven dimensions. We compare the ATLAS9/ASS $\epsilon$ T and MARCS/Turbospectrum libraries and apply both of them to the analysis of the observed H-band spectra of the Sun and the K2 giant Arcturus, as well as to a selected sample of well-known giant stars observed at very high resolution. The new APOGEE libraries are publicly available and can be employed for chemical studies in the H-band using other high-resolution spectrographs.
\end{abstract}

Key words: astrochemistry - radiative transfer - stars: atmospheres

\section{INTRODUCTION}

Previous near-infrared (JHK bands) spectroscopic observations of individual or small selected samples of giant stars have been limited in scope and mostly biased toward the brightest sources. This has prevented the study of the chemical abundance patterns in unbiased samples of stars toward the inner (and dusty) parts (e.g., Galactic bulge and center) of our Galaxy, significantly hampering progress toward a full understanding of the formation and chemical (and dynamical) evolution of the Milky Way. This unfortunate situation has dramatically changed in the new era of massive high-resolution spectroscopic surveys. In particular, the Apache Point Observatory Galactic Evolution Experiment (APOGEE) has focused on collecting high-resolution and high-quality H-band spectra for a large $\left(>10^{5}\right.$ stars $)$ sample of giant stars, with access to the inner-and more extinguished-regions of our Galaxy.

APOGEE is one of the four spectroscopic surveys of the Sloan Digital Sky Survey-III (SDSS-III; e.g., Eisenstein et al. 2011). It is a high-resolution $(R \equiv \lambda / \Delta \lambda=22,500)$
H-band (1.514-1.696 $\mu \mathrm{m})$ spectroscopic survey spanning all stellar populations in our Galaxy (see e.g., Allende Prieto et al. 2008; Eisenstein et al. 2011; Majewski et al. 2015). During the period from 2011 to 2014, the APOGEE survey collected about 500,000 spectra of $\sim 146,000$ stars, predominantly post-main-sequence stars (red giants, subgiants, and red clump stars), using the Sloan Foundation $2.5 \mathrm{~m}$ telescope (Gunn et al. 2006) and an innovative multi-object IR spectrograph (Wilson et al. 2010). High-resolution stellar spectra of red giants in the H-band show a rich diversity of absorption lines from a wide variety of atoms and molecules, with $\mathrm{OH}, \mathrm{CN}$, and $\mathrm{CO}$ the most important molecular contributors. To ascertain the stellar atmospheric parameters and measure chemical abundances from the observed spectra, the APOGEE Atmospheric Stellar Parameters and Chemical Abundances Pipeline (ASPCAP; García Pérez et al. 2015) relies on an algorithm that identifies the best-fitting synthetic spectrum for each observed spectrum. The fitting code uses interpolation over pre-computed multi-dimensional grids of synthetic spectra (i.e., model stellar spectral libraries) to find 
the best model (with the minimum $\chi^{2}$ values) for each observed spectrum. Synthetic spectra are calculated using classical model atmospheres (see, e.g., Mészáros et al. 2012) and extensive atomic and molecular line lists (Shetrone et al. 2015).

The SDSS-III APOGEE public data release 10 (DR10; Ahn et al. 2014) was based on synthetic spectral libraries computed using Castelli \& Kurucz (2003) model atmospheres (see, e.g., Mészáros et al. 2013). The Castelli \& Kurucz (2003) model atmospheres incorporate line opacity by means of opacity distribution functions (ODFs) and are based on solar (or scaled solar) chemical abundances from Grevesse \& Sauval (1998). For the APOGEE main survey targets, the fundamental stellar parameters (effective temperature $\left(T_{\text {eff }}\right)$, surface gravity $(\log g)$, and metallicity $([\mathrm{M} / \mathrm{H}]))^{18}$ and the relative abundances of $\alpha$ elements $([\alpha / \mathrm{M}]$; in this case $\mathrm{O}, \mathrm{Mg}, \mathrm{Si}, \mathrm{S}, \mathrm{Ca}$, and $\mathrm{Ti})$, carbon $([\mathrm{C} / \mathrm{M}])$, and nitrogen $([\mathrm{N} / \mathrm{M}])$ were released in DR10. The final SDSS-III APOGEE public data release, DR12 (Alam et al. 2015) is based on self-consistent spectral libraries, where the same chemical abundances are used both in the computation of the model atmospheres and in the spectral synthesis. Moreover, newer solar reference abundances from Asplund et al. (2005) are now adopted. ${ }^{19}$ In addition to the main stellar parameters the individual element abundances for up to 15 elements (typically with a precision of 0.1 dex or better) are also released in DR12.

In this paper we present for the first time the H-band stellar spectral libraries for the SDSS-III APOGEE survey, ${ }^{20}$ which can be used as well for chemical studies using other highresolution spectrographs working in the H-band. The DR12 spectral libraries are based on ATLAS9 model atmospheres (see Mészáros et al. 2012) and calculated with the ASS $\epsilon$ T (Koesterke et al. 2008; Koesterke 2009) spectral synthesis code. In addition to the official family of ATLAS9/ASS $\epsilon$ T DR12 spectral libraries, we have computed similar spectral libraries based on MARCS model atmospheres, with the Turbospectrum synthesis code (Alvarez \& Plez 1998; Plez 2012). We provide a comparison between these different model atmospheres and spectral synthesis codes in order to check the validity of the adopted DR12 synthetic spectral libraries. In Section 2, we describe the parameter range and the calculation method of the ATLAS9/ASS $\epsilon$ T and MARCS/ Turbospectrum stellar spectral libraries, while Section 3 discusses systematic differences between the two grids of synthetic spectra. Both grids are applied to the observed Hband spectra of the Sun and the K2 giant Arcturus in Section 4. In Section 5, we use both model stellar spectral libraries to derive the chemical patterns in a selected sample of wellknown giant stars observed with the Fourier Transform Spectrograph on the Kitt Peak National Observatory $4 \mathrm{~m}$ Mayall reflector. Our main conclusions and future work are given in Section 6.

\footnotetext{
18 A linear relationship between microturbulence and surface gravity is adopted (see García Pérez et al. 2015); e.g., $\xi=2.478-0.325 \times \log g$ is used in DR12 (Alam et al. 2015)

19 The helium reference abundance adopted for the computation of the synthetic spectral libraries is $12+\log _{10}\left(N_{\mathrm{He}} / N_{\mathrm{H}}\right)=10.93$, where $N_{\mathrm{He}}$ and $N_{\mathrm{H}}$ are the number density of helium and hydrogen nuclei, respectively.

${ }^{20}$ The stellar spectral libraries are available online; data.sdss 3.org/sas/dr12/ apogee/spectro/redux/speclib/asset/kurucz_filled/solarisotopes/.
}

\section{SYNTHETIC SPECTRAL LIBRARIES FOR APOGEE}

As mentioned above, the SDSS-III APOGEE DR12 results (Alam et al. 2015) are based on ATLAS9/ASS $e$ T synthetic spectral libraries. The APOGEE synthetic spectral libraries are continuously improved and will be updated in the future (see Section 6). In this section, we describe two H-band stellar spectral libraries developed for the SDSS-III APOGEE survey, based on ATLAS9 and MARCS model atmospheres and computed with the ASS $\epsilon$ T and Turbospectrum spectral synthesis codes, respectively. All MARCS/Turbospectrum computations were performed on the Condor cluster at the Instituto de Astrofísica de Canarias (IAC). Condor (or HTCondor) is a High Throughput Computing system developed by the University of Wisconsin-Madison. ${ }^{21}$ In the case of the IAC, Condor consists of a computer cluster with 808 CPUs. On the other hand, the ATLAS9/ASS $\epsilon$ T calculations were performed on the clusters Stampede and Maverick operated by the Texas Advanced Computing Center.

It is to be noted here that the ATLAS9 and MARCS model atmospheres do not include the line opacity for the polyatomic carbon molecules $\mathrm{C}_{2} \mathrm{H}_{2}$ and $\mathrm{C}_{3}$ (Mészáros et al. 2012). These molecules are known to dominate the infrared spectra of cool $\left(T_{\text {eff }}<4000 \mathrm{~K}\right)$ carbon stars with $\mathrm{C} / \mathrm{O}>1.0$, strongly affecting their thermal atmospheric structure. Thus, at present the ATLAS9/ASS $\epsilon$ T and MARCS/Turbospectrum synthetic spectra with $T_{\text {eff }}<3500-4000 \mathrm{~K}$ and $\mathrm{C} / \mathrm{O}>1.0$ are not reliable (see Section 6).

\subsection{ATLAS9/ASSET Spectral Library}

The APOGEE ATLAS9/ASS $\epsilon$ T spectral library makes use of the new ATLAS9 grid of model atmospheres presented in Mészáros et al. (2012). ATLAS9 model atmospheres (Kurucz 1993) are one-dimensional plane-parallel models computed in LTE and using opacity ODFs to handle line opacity (see, e.g., Kurucz 2005). The mixing-length scheme for convective energy transport is adopted, and the Kurucz atomic and molecular line lists ${ }^{22}$ are used (see Mészáros et al. 2012 for more details).

The new ATLAS9 model atmospheres ${ }^{23}$ are based on the recent solar composition by Asplund et al. (2005). This has the advantage of matching the solar composition adopted also in the construction of the MARCS models described in the next section. Mészáros et al. (2012) carried out new ODFs and Rosseland mean opacity calculations for several microturbulent velocities $\left(\xi=0,1,2,4\right.$, and $\left.8 \mathrm{~km} \mathrm{~s}^{-1}\right)$, but all the APOGEE ATLAS9 model atmospheres are computed with $\xi=2 \mathrm{~km} \mathrm{~s}^{-1}$.

For the purpose of building the libraries, we divided the ATLAS9 model atmospheres into two grids (GK and F) depending on the effective temperature $\left(T_{\text {eff }}\right)$, with the GK- and F-classes covering the $3500-6500 \mathrm{~K}$ and $5500-8000 \mathrm{~K} T_{\text {eff }}$ ranges, respectively. It is important to note that missing (nonconverged) ATLAS9 model atmospheres are substituted by the nearest model in chemical space at the same $T_{\text {eff }}$ and $\log g$. The non-converged ATLAS9 model atmospheres were a total of 6217 structures, most of them corresponding tocool high surface gravity models $\left(T_{\text {eff }}<4000 \mathrm{~K}\right.$ and $\left.\log g>4.0\right)$ and cool C-rich models $\left(T_{\text {eff }}<4000 \mathrm{~K}\right.$ and $\left.\mathrm{C} / \mathrm{O}>0.75\right)$.

\footnotetext{
21 http://research.cs.wisc.edu/htcondor/

$22 \mathrm{http}: / /$ kurucz.harvard.edu/linelists.html

$23 \mathrm{http}: / / \mathrm{www}$.iac.es/proyecto/ATLAS-APOGEE/
} 
Table 1

Parameters of the ATLAS9/ASS $\epsilon$ T and MARCS/Turbospectrum Spectral Libraries

\begin{tabular}{|c|c|c|c|c|c|c|c|}
\hline Class & $\begin{array}{c}T_{\text {eff }}^{\text {range }(\text { step })} \\
(\mathrm{K})\end{array}$ & $\begin{array}{c}\log g \\
\text { range (step) } \\
\quad(\operatorname{dex})\end{array}$ & $\begin{array}{c}{[\mathrm{M} / \mathrm{H}]} \\
\text { range }(\text { step}) \\
(\mathrm{dex})\end{array}$ & $\begin{array}{c}{[\mathrm{C} / \mathrm{M}]} \\
\text { range }(\text { step}) \\
(\mathrm{dex})\end{array}$ & $\begin{array}{c}{[\mathrm{N} / \mathrm{M}]} \\
\text { range }(\text { step }) \\
(\mathrm{dex})\end{array}$ & $\begin{array}{c}{[\alpha / \mathbf{M}]} \\
\text { range }(\text { step }) \\
(\operatorname{dex})\end{array}$ & $\begin{array}{c}\log \xi \text { range (step })^{\mathrm{a}} \\
\left(\mathrm{km} \mathrm{s}^{-1}\right)\end{array}$ \\
\hline \multicolumn{8}{|c|}{ ATLAS9/ASS $\epsilon$ T } \\
\hline GK & $3500-6000(250)$ & $0-5(0.5)$ & $-2.5-0.5(0.5)$ & $\begin{array}{c}-1.0-1.0 \\
(0.25)\end{array}$ & $-1.0-1.0(0.5)$ & $\begin{array}{c}-1.0-1.0 \\
(0.25)\end{array}$ & $\begin{array}{c}-0.301-0.903 \\
(0.301)\end{array}$ \\
\hline $\mathrm{F}$ & $5500-8000(250)$ & $0-5(0.5)$ & $-2.5-0.5(0.5)$ & $\begin{array}{c}-1.0-1.0 \\
(0.25)\end{array}$ & $-1.0-1.0(0.5)$ & $\begin{array}{c}-1.0-1.0 \\
(0.25)\end{array}$ & $\begin{array}{c}-0.301-0.903 \\
(0.301)\end{array}$ \\
\hline \multicolumn{8}{|c|}{ MARCS/Turbospectrum } \\
\hline $\mathrm{K}$ & $3500-5500(250)$ & $0-5(0.5)$ & $-2.5-0.5(0.5)$ & $-1.0-1.0(0.5)$ & $-1.0-1.0(0.5)$ & $-1.0-1.0(0.5)$ & $\begin{array}{c}-0.301-0.903 \\
(0.301)\end{array}$ \\
\hline
\end{tabular}

a The microturbulence $(\log \xi)$ step is given in $\log _{10}$ units (uniform step of $0.301 \mathrm{dex}$ ).

Most stars targeted by SDSS-III APOGEE are red giant and dwarf candidates, which makes the ATLAS9 GK-class model grid the most important for APOGEE. The ATLAS9 GK-class models cover eleven $T_{\text {eff }}$ and eleven $\log g$ values (from 3500 to $6000 \mathrm{~K}$ in steps of $250 \mathrm{~K}$ and from 0 to $5 \mathrm{dex}$ in steps of 0.5 dex, respectively), seven metallicities $[\mathrm{M} / \mathrm{H}]^{24}$ (from -2.5 to $+0.5 \mathrm{dex}$ in steps of $0.5 \mathrm{dex})$, and 81 values for the abundance of $\mathrm{C}$ and the $\alpha$-elements $([\mathrm{C} / \mathrm{M}]$ and $[\alpha / \mathrm{M}]$ from -1.0 to $+1.0 \mathrm{dex}$ in steps of $0.25 \mathrm{dex})$. The $\alpha$-elements in the ATLAS9 models areas in the introduction. Thus, 68,607 GKclass ATLAS9 models are present in the GK ATLAS9 APOGEE grid used to produce the ATLAS9/ASS $\epsilon$ T spectral library.

The ATLAS9 F-class grid is used for the analysis of a much smaller number of warmer stars such as the telluric line standards observed by the SDSS-III APOGEE survey. The Fclass ATLAS9-APOGEE grid also contains 68,607 model atmospheres that cover $T_{\text {eff }}$ values from $5500 \mathrm{~K}$ to $8000 \mathrm{~K}$ (in steps of $250 \mathrm{~K})$ and the same $[\mathrm{M} / \mathrm{H}],[\mathrm{C} / \mathrm{M}]$, and $[\alpha / \mathrm{M}]$ ranges as the GK-class one.

The code ASS $\epsilon$ T was used to compute synthetic spectra for the Kurucz ATLAS9 models. ASS $\epsilon$ T was originally written for calculating spectra for 3D hydrodynamical simulations, and later a 1D branch was developed for dealing with large numbers of classical hydrostatic model atmospheres. The code has an option to pre-calculate the opacity as a function of temperature and a second thermodynamical quantity (density or electron density) on a grid covering the range of the models of interest, interpolating on-the-fly for solving the radiative transfer equation. Opacity interpolations were avoided in the calculations described above in order to maximize accuracy. ASS $\epsilon$ T adopts the same software package used by Synspec/ Tlusty (Hubeny \& Lanz 1995; Hubeny 2006) for computing opacities, and the input data described by Allende Prieto (2008). Continuum opacities are mainly from the Opacity Project (Cunto et al. 1993) and the Iron Project (Nahar 1995; Bautista 1997), and line opacities are shared in the calculations described in this paper for Turbospectrum/MARCS.

\footnotetext{
${ }^{24}$ The overall metallicity $[\mathrm{M} / \mathrm{H}]$ accounts for all elements with atom number $\mathrm{Z}>2$ and $[\mathrm{M} / \mathrm{H}]=\log _{10}\left(N_{\mathrm{M}} / N_{\mathrm{H}}\right)_{\star}-\log _{10}\left(N_{\mathrm{M}} / N_{\mathrm{H}}\right)_{\odot}$, where $N_{\mathrm{M}}$ and $N_{\mathrm{H}}$ are the number density of any $Z>2$ element and hydrogen nuclei, respectively. For the construction of the synthetic spectral libraries, all metals other than $\mathrm{C}, \mathrm{N}$, and $\alpha$-elements are scaled with the corresponding $[\mathrm{M} / \mathrm{H}]$ value.
}

The opacities used in ASS $\epsilon$ T are largely independent from those in Kurucz's model atmospheres, especially in the case of continuum (bound-free and free-free) opacity. We have, nevertheless, checked that the absolute fluxes predicted in the optical and near-infrared are fairly similar for ASS $\epsilon$ T and ATLAS9 for a solar-like star, where $\mathrm{H}^{-}$dominates the continuum opacity (as for most APOGEE targets), while in the near-UV the differences become larger due to different photoionization cross-sections for metals (mainly iron, but also magnesium and other atoms). The overall equation of state is also similar for the two codes, since when chemical equilibrium calculations are performed in $\mathrm{ASS} \epsilon \mathrm{T}$, the final electron density for solar-like stars is consistent with the original from the ATLAS9 model at a level of a few percent. In all the ATLAS9 DR12 grids the electron density was iterated in ASS $\epsilon$ T to be consistent with the atomic and molecular species considered in the synthesis. The calculations for a few models with extreme compositions did result in iterated electron densities that shifted the $\tau_{\lambda} \sim 100$ layer outside of the ATLAS9 structure. ASS $\epsilon$ T is set to stop when that occurs, so those calculations were successfully completed relaxing that limit to $\tau_{\lambda} \sim 10$.

The ATLAS9 APOGEE GK- and F-class model atmosphere grids mentioned above are used in conjunction with the DR12 atomic/molecular line lists (linelist 201312161124; Shetrone et al. 2015) to compute ATLAS9/ASS $\epsilon$ T synthetic spectra with five microturbulence values $\left(\xi=0.5,1,2,4\right.$, and $\left.8 \mathrm{~km} \mathrm{~s}^{-1}\right)$, five $\mathrm{N}$ abundances $([\mathrm{N} / \mathrm{M}]$ from -1.0 to +1.0 dex in steps of $0.5 \mathrm{dex})$, and the solar $\mathrm{C}$ isotopic ratio $\left({ }^{12} \mathrm{C} /{ }^{13} \mathrm{C}=89\right)$. Thus, in the end the GK- and F-class ATLAS9/ASS $\epsilon$ T grids contain seven dimensions $\left(7 \mathrm{D} ; T_{\text {eff }}, \log g, \xi,[\mathrm{M} / \mathrm{H}],[\mathrm{C} / \mathrm{M}],[\mathrm{N} / \mathrm{M}]\right.$, and $[\alpha / \mathrm{M}])$ and the same number $(1,715,175)$ of total synthetic spectra. The model parameters for the ATLAS9/ASS $\epsilon$ T stellar spectral libraries are summarized in Table 1. Finally, the synthetic spectra are smoothed to the APOGEE resolution, continuum-normalized, re-sampled, and transformed to vacuum wavelengths. The ATLAS9/ASS $\epsilon$ T spectral libraries are then compressed using Principal Component Analysis method (PCA; Pearson 1901) to prepare it for use by ASPCAP (see García Pérez et al. 2015 for further details). We note that the line spread function (LSF) for the ATLAS9/ASS $\epsilon$ T DR12 spectral library is a combo of those derived from several APOGEE fibers, actually an average of five fiber LSFs characterized with a Gauss-Hermite function of variable resolution with wavelength (see also García Pérez et al. 2015 
for further details). However, to make a fair comparison with the other spectral libraries treated in this paper and to avoid also systematic effects, we are using an ATLAS9/ASS $\epsilon$ T spectral library version that has been smoothed with a Gaussian kernel to the APOGEE spectral resolution $(R=22,500)$, as the MARCS/Turbospectrum one.

\subsection{MARCS/Turbospectrum Spectral Library}

The APOGEE MARCS/Turbospectrum spectral library is based on the most recent MARCS model atmospheres (Gustafsson et al. 2008). Basically, MARCS model atmospheres are one-dimensional models in hydrostatic equilibrium that are calculated in LTE and adopting the mixing-length theory for convection (Henyey et al. 1965). The MARCS models are spherical for surface gravities $\log g \leqslant 3$, while they are plane-parallel at higher gravities (appropriate for dwarf stars; see Gustafsson et al. 2008). Line opacities are treated with opacity sampling (OS).

The grid of MARCS model atmospheres for APOGEE was presented in Mészáros et al. (2012) ${ }^{25}$ and we refer the reader to this paper and Gustafsson et al. (2008) for more details. MARCS models covering $9 T_{\text {eff }}$ values (from 3500 to $5500 \mathrm{~K}$ in steps of $250 \mathrm{~K}$ ), ${ }^{26} 11 \log g$ values (from 0 to 5 dex in steps of $0.5 \mathrm{dex}$ ), ${ }^{27} 7$ metallicities $[\mathrm{M} / \mathrm{H}]$ (from -2.5 to $+0.5 \mathrm{dex}$ in steps of $0.5 \mathrm{dex}$ ), and 25 combinations of $\mathrm{C}$ and $\alpha$-element abundances $^{28}([\mathrm{C} / \mathrm{M}]$ and $[\alpha / \mathrm{M}]$ from -1.0 to +1.0 dex in steps of $0.5 \mathrm{dex}$ ) are present in the MARCS-APOGEE grid used to construct the corresponding MARCS/Turbospectrum spectral library. This results in a grid of $\sim 17,325$ MARCS models; 1062 models, however, do not converge and are therefore missing in the MARCS-APOGEE grid. These non-converged MARCS model atmosphere structures are replaced with the nearest model in chemical space at the same $T_{\text {eff }}$ and $\log g$ (see below).

Synthetic spectra in the APOGEE spectral range were generated with the Turbospectrum package (Alvarez \& Plez 1998; Plez 2012), which shares the same input data and radiative transfer routines with MARCS (Gustafsson et al. 2008). Turbospectrum is a 1D LTE spectral synthesis code that accounts for 600 molecules and uses the treatment of collisional line broadening described by Anstee \& O'Mara (1995) and Barklem et al. (2000). It allows the computation of flux (or intensity) synthetic spectra for stars of spectral type $\mathrm{F}$ and cooler. We used version 13.1 of Turbospectrum but modified by us to use van der Waals broadening (the Barklem treatment was used when possible, otherwise we used van der Waals constants from Kurucz; see Shetrone et al. 2015 for more details) for the atomic lines. ${ }^{29}$ The synthetic spectra are computed for an array of standard air wavelengths with a wavelength step of $0.03 \AA$. By using the MARCS-APOGEE grid (see above) and the DR12 atomic/molecular line lists (Shetrone et al. 2015) as input in Turbospectrum, we

\footnotetext{
25 The MARCS model atmospheres can be found in the MARCS Web site: http://marcs.astro.uu.se/.

${ }^{26}$ We note that the MARCS models with $T_{\text {eff }}=3700 \mathrm{~K}$ are used instead of those with $3750 \mathrm{~K}$, because the $T_{\text {eff }}$ step in the MARCS grid is $100 \mathrm{~K}$ below $4000 \mathrm{~K}$.

27 Note that plane-parallel and spherical MARCS model atmospheres are computed with a microturbulence $(\xi)$ of 1 and $2 \mathrm{~km} \mathrm{~s}^{-1}$, respectively (see, e.g., Mészáros et al. 2012).

28 The $\alpha$-elements in MARCS are $\mathrm{O}, \mathrm{Ne}, \mathrm{Mg}, \mathrm{Si}, \mathrm{S}, \mathrm{Ar}, \mathrm{Ca}$, and Ti.

29 Version 14.1 of Turbospectrum includes these changes and it is publicly available at http://ascl.net/1205.004.
}

constructed MARCS/Turbospectrum synthetic spectra with microturbulent velocities of $\xi=0.5,1,2,4$, and $8 \mathrm{~km} \mathrm{~s}^{-1}$ and with varying $\mathrm{N}$ content $([\mathrm{N} / \mathrm{M}]$ from -1.0 to +1.0 in steps of $0.5 \mathrm{dex}$ ) and solar ${ }^{12} \mathrm{C} /{ }^{13} \mathrm{C}$ ratio. This resulted in a MARCS/ Turbospectrum grid with seven dimensions $\left(7 \mathrm{D} ; T_{\mathrm{eff}}, \log g, \xi\right.$, $[\mathrm{M} / \mathrm{H}],[\mathrm{C} / \mathrm{M}],[\mathrm{N} / \mathrm{M}]$, and $[\alpha / \mathrm{M}])$ containing $~ 43,375$ synthetic spectra; the model parameters for the MARCS/Turbospectrum stellar spectral library are also given in Table 1 . It is to be noted here that in some cases $(\sim 100)$ the spectral synthesis does not converge. However, these mainly correspond to the most extreme and unrealistic abundance patterns (e.g., very high or very low $\alpha$ element abundances). As in the case of the missing MARCS model atmospheres mentioned above, we also replace the missing synthetic spectra (fluxes) with the ones at the same $T_{\text {eff }}$ and $\log g$ and with the nearest chemical composition. Finally, the MARCS/Turbospectrum synthetic spectra are processed (i.e., smoothed, continuum-normalized, etc.) and PCA-compressed in the same way as the ATLAS9/ASS $\epsilon$ T library.

\section{COMPARISON BETWEEN ATLAS9/ASS $\epsilon$ T-MARCS/ TURBOSPECTRUM SPECTRAL LIBRARIES}

In this section we compare the Gaussian smoothed GKATLAS9/ASS $\epsilon$ T and the K-MARCS/Turbospectrum spectral libraries, which since these grids overlap in atmospheric parameters in the range $3500-5500 \mathrm{~K}$.

Such comparisons can give indications on the uncertainties due to the use of different model atmospheres, different spectral synthesis codes, as well as partly different input data, with only the line lists being the same. It also allows to check the possible influence of sphericity effects in the synthetic spectra (Section 3.1) (30 $^{30}$ and to explore possible systematic differences between both synthetic libraries in the 7D parameter space (Section 3.2).

A total of 2552 GK-ATLAS9/ASS $\epsilon$ T test synthetic spectra were chosen to uniformly span the parameter space, while avoiding the grid boundaries; e.g., by selecting one in ten consecutive spectra with different 7D parameters in the nodes of the grid. Our synthetic spectra have surface gravities from $\log g=0.5$ to 4.5 dex, effective temperatures $T_{\text {eff }}$ from 3750 to $5250 \mathrm{~K}$, microturbulent velocities from 1 to $4 \mathrm{~km} \mathrm{~s}^{-1},[\mathrm{C}, \mathrm{N}, \alpha /$ $\mathrm{M}]$ from -0.5 to $0.5 \mathrm{dex}$, and $[\mathrm{M} / \mathrm{H}]$ from -2.0 to $0.0 \mathrm{dex}$.

\subsection{Comparisons between Spectral Syntheses}

For the study of possibledifferences (e.g., sphericity effects) between both families of synthetic spectra, we extracted the same 2,552 test synthetic spectra from the K-MARCS/ Turbospectrum spectral library. The differences in the synthetic spectra are estimated by deriving the rms difference between the GK-ATLAS9/ASS $\epsilon$ T and the K-MARCS/Turbospectrum pair of spectral syntheses with the same 7D parameters. We performed comparisons for two groups: low-gravity stars (log $g \leqslant 2.0)$ and high-gravity stars $(\log g \geqslant 3.0)$. In this way, if sphericity effects are affecting the H-band synthetic spectra of the low-gravity stars, we would find higher rms values for this group.

Our results are summarized in Figures 1 and 2, where we compare the rms values from some synthetic spectra in our test sample; i.e., those with $\log g \leqslant 2.0,[\mathrm{C} / \mathrm{M}]=0.0,[\mathrm{~N} / \mathrm{M}]=0.0$,

\footnotetext{
${ }^{30}$ Sphericity effects are expected to be noticeable for low-gravity stars, as all ATLAS9 model atmospheres have plane-parallel geometry.
} 
ATLAS9/ASSET vs. MARCS/Turbospectrum synthetic spectra

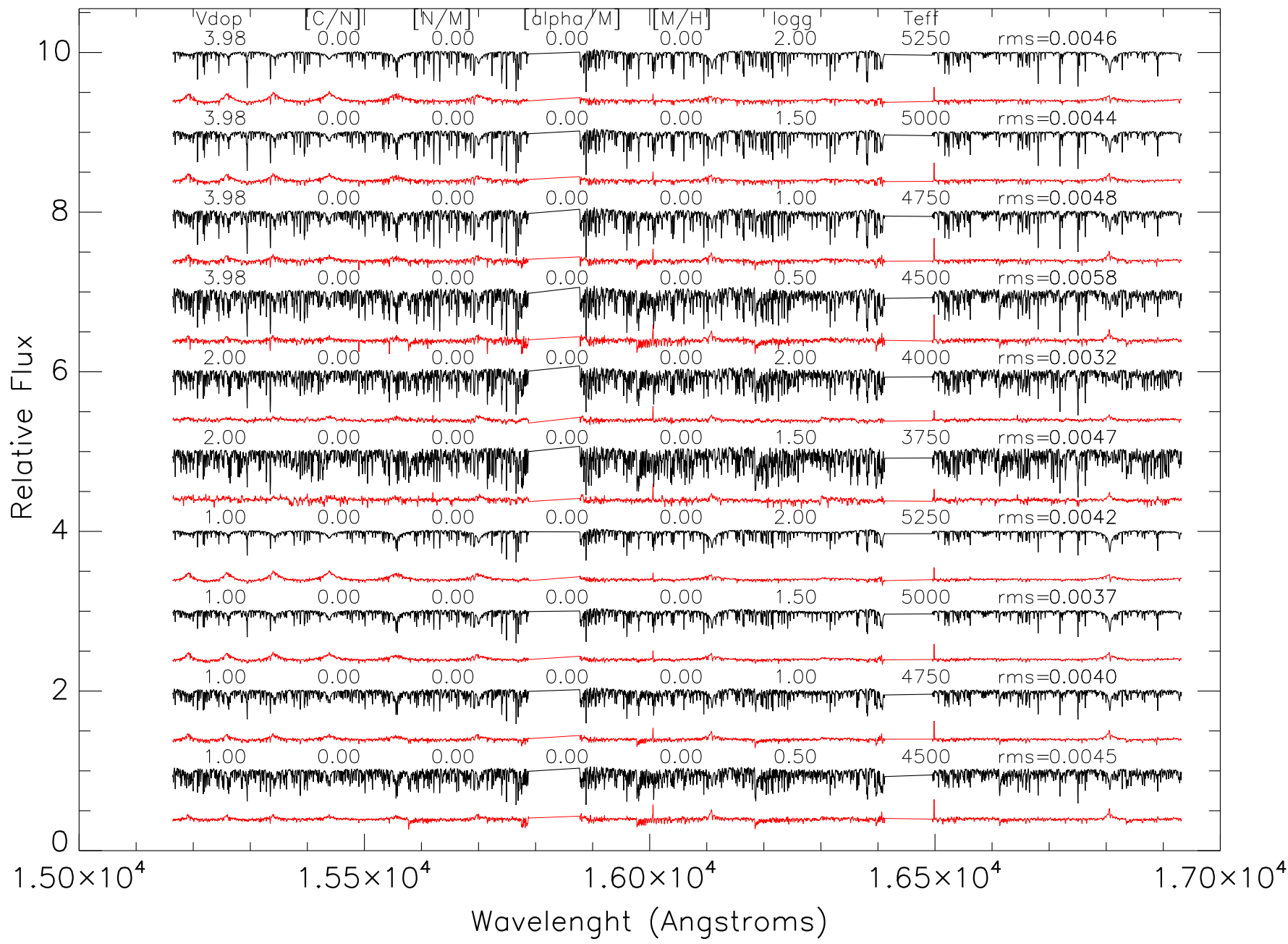

Figure 1. Comparison between synthetic spectra extracted from the GK-ATLAS9/ASS $\epsilon$ and K-MARCS/Turbospectrum libraries for low-gravity stars $(\log g \leqslant 2$ ). The wavelength ranges covered by the three APOGEE detectors are showed. Only ATLAS9/ASS $\epsilon$ T spectra are displayed (in black). The residuals, computed as MARCS-ATLAS9 fluxes, have been multiplied by a factor of five to make them visible in the figure (red line). The 7D parameters of each spectra are indicated above each spectrum (where $\xi \equiv \mathrm{v}_{\mathrm{dop}}$ ), together with the rms value computed from each pair of synthetic spectra with the same 7D parameters.

$[\alpha / \mathrm{M}]=0.0$ and $[\mathrm{M} / \mathrm{H}]=0.0$ (Figure 1 , showing 10 synthetic spectra in our test sample) and those with $\log g \geqslant 3.0$, $[\mathrm{C} / \mathrm{M}]=0.0,[\mathrm{~N} / \mathrm{M}]=0.0,[\alpha / \mathrm{M}]=0.0$ and $[\mathrm{M} / \mathrm{H}]=0.0$ (Figure 2, displaying 7 synthetic spectra in our test sample). The rms values (of the order of $\sim 0.3 \%-0.6 \%$ ) are very similar for the two groups. Interestingly, this indicates that the overall sphericity effects on the chemical abundances are smaller in the H-band than in the optical wavelength region (Heiter \& Eriksson 2006 found abundance differences as high as $0.35 \mathrm{dex}$ ); one reason may be that in the H-band we observe deeper atmospheric layers. Indeed, we obtain similar rms values for other chemical compositions $([\mathrm{C} / \mathrm{M}],[\mathrm{N} / \mathrm{M}],[\alpha / \mathrm{M}]$, and $[\mathrm{M} / \mathrm{H}]$ values), validating the adoption of our official DR12 library based on ATLAS9 models without any significant biases due to the use of plane-parallel model atmospheres. The low rms values obtained are indicative of no large differences between the computations based on ASS $\epsilon$ T and Turbospectrum spectral synthesis codes. We also note that the (systematically) deviating features in Figures 1 and 2 are hydrogen lines; this is because the spectral synthesis codes (ASS $\epsilon$ T and Turbospectrum) use different internal data for $\mathrm{H}$. However, in the following section the $\mathrm{H}$ lines are not used in ASPCAP infitting the best-fit spectra.

\subsection{Systematic Differences}

With the ultimate goal of exploring further the possible systematic differences between both grids of APOGEE synthetic spectra, the 2552 synthetic spectra in our test sample, as extracted from the GK-ATLAS9/ASS $\epsilon$ T spectral library, have been fitted with the K-MARCS/Turbospectrum library using ASPCAP (see García Pérez et al. 2015). In other words, we find out what MARCS/Turbospectrum seven (7D) stellar parameters are recovered by the pipeline when we treat the GKATLAS9/ASS $\epsilon$ T synthetic spectra as input observed spectra.

The results of this exercise are reported in Table 2 and in Figures 3 and 4. In Table 2 (columns 2 and 3) we show the median in the difference $\Delta$ (=output MARCS parameterinput ATLAS9 parameter) and the dispersion $(\sigma)$ of the differences obtained for the full sample. To avoid outliers from biasing the statistics, $\sigma$ is computed as the difference between the maximum and minimum $\Delta$ after excluding the largest $15.85 \%$ of the sample and the smallest $15.85 \%$, and divide it by two, which would correspond to the standard deviation in a Normal distribution (see Figure 3). The results of fitting the 2552 test synthetic spectra with the GK-ATLAS9/ASS $\epsilon$ T library are displayed in Table 2 (columns 4 and 5) and 
ATLAS9/ASSET VS. MARCS/Turbospectrum synthetic spectra

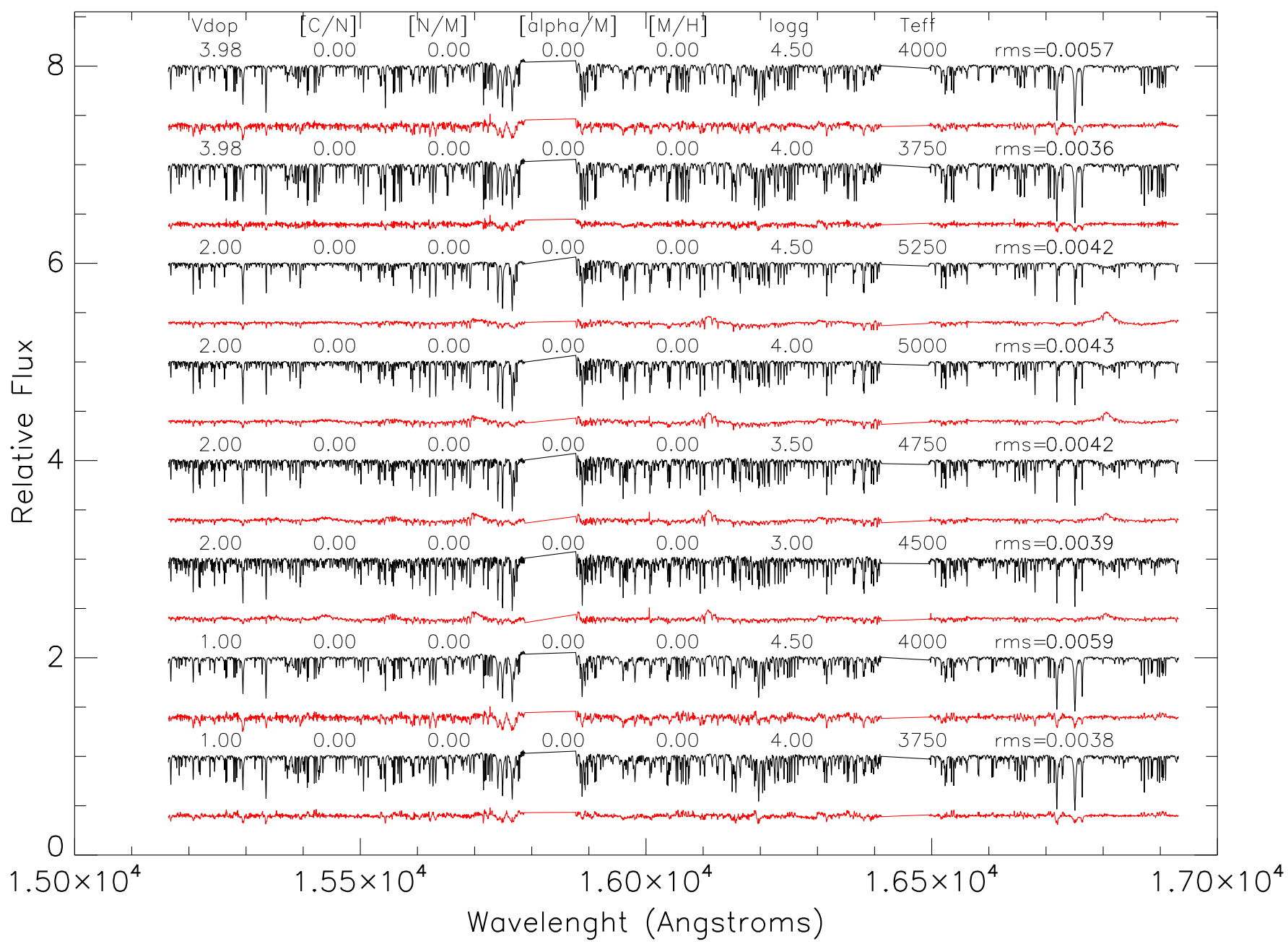

Figure 2. Same as Figure 1, but for $\log g \geqslant 3.0$.

Figure 4. In addition, we compare the ATLAS9 versus MARCS 7D parameters for two different sub-samples: lowgravity stars $(\log g \leqslant 2.0$. $)$ and high-gravity stars $(\log g \geqslant 3.0$; see Table 2, columns 6-9).

The MARCS/Turbospectrum spectral library (full sample) provides slightly higher effective temperature, microturbulence, $[\mathrm{N} / \mathrm{M}]$, and $[\alpha / \mathrm{M}]$, with median values of $38.1 \mathrm{~K}, 0.02,0.09$, and 0.02 dex, respectively. On the other hand, the MARCS/
Turbospectrum library provides slightly lower metallicity and surface gravity than the ATLAS9/ASS $\epsilon$ T one; median values of -0.03 and -0.13 dex are found for $[\mathrm{M} / \mathrm{H}]$ and $\log g$, respectively. Very similar carbon abundances $([\mathrm{C} / \mathrm{M}])$ are obtained with the MARCS/Turbospectrum spectral grid. In order to check if the differences in the 7D parameters mentioned above are significant or merely the results of degeneracies, we compare the previous

Table 2

Systematic Differences between ATLAS9/ASS $\epsilon$ T (Input) and MARCS/Turbospectrum (Output) Spectral Syntheses in the 7D Parameter Space

\begin{tabular}{|c|c|c|c|c|c|c|c|c|}
\hline Parameter & $\begin{array}{c}\text { Median(MARCS- } \\
\text { ATLAS9) } \\
\text { Full sample }\end{array}$ & $\sigma$ & $\begin{array}{c}\text { Median(ATLAS9- } \\
\text { ATLAS9) }^{\mathrm{a}} \\
\text { Full sample }\end{array}$ & $\sigma$ & $\begin{array}{c}\text { Median(MARCS- } \\
\text { ATLAS9) } \\
\log g \leqslant 2.0 \text { subsample }\end{array}$ & $\sigma$ & $\begin{array}{c}\text { Median(MARCS- } \\
\text { ATLAS9) } \\
\log g \geqslant 3.0 \text { subsample }\end{array}$ & $\sigma$ \\
\hline $\log \xi$ & 0.02 & 0.08 & 0.00 & 0.03 & 0.01 & 0.02 & 0.08 & 0.19 \\
\hline$[\mathrm{N} / \mathrm{M}]$ & 0.09 & 0.20 & 0.01 & 0.15 & 0.10 & 0.17 & 0.07 & 0.27 \\
\hline$[\alpha / \mathrm{M}]$ & 0.02 & 0.02 & 0.00 & 0.01 & 0.03 & 0.02 & 0.01 & 0.03 \\
\hline$[\mathrm{M} / \mathrm{H}]$ & -0.03 & 0.04 & 0.00 & 0.01 & 0.01 & 0.04 & -0.04 & 0.04 \\
\hline
\end{tabular}

a These are the results using synthetic spectra from the ATLAS9/ASS $\epsilon$ T library as input and output for ASPCAP. 

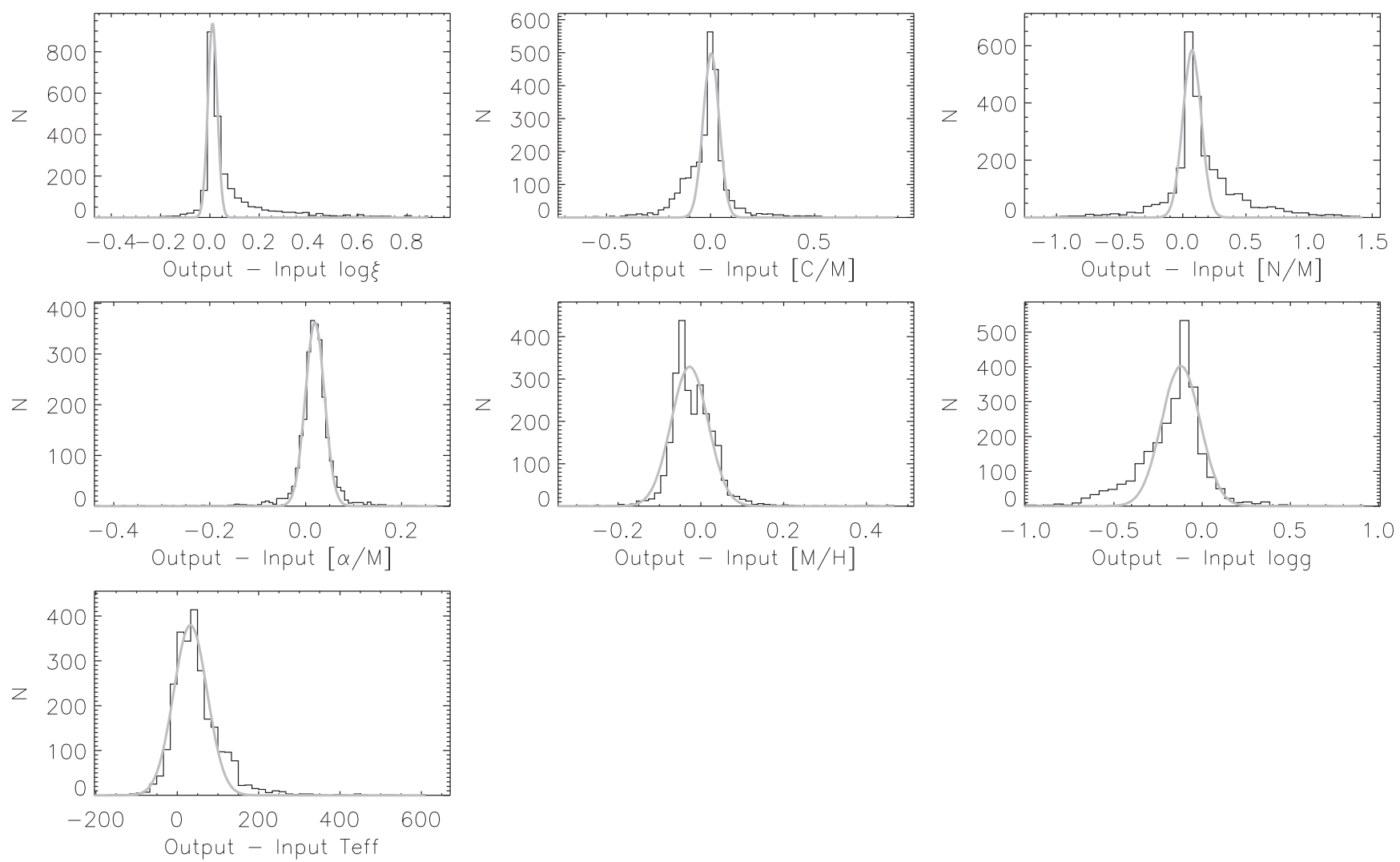

Figure 3. Systematic differences between the ATLAS9/ASS $\epsilon$ T and the MARCS/Turbospectrum spectral libraries in the 7D parameter space. The input stellar parameters are those from the ATLAS9/ASS $\epsilon$ T spectral library, while the output parameters are those derived/recovered with the MARCS/Turbospectrum library by using ASPCAP. The distribution of the differences (i.e., output MARCS parameter-input ATLAS9 parameter) is shown for the 7D grid (in black). A Gaussian distribution fit, excluding the $\sim 15.85 \%$ of the largest data differences (i.e., outliers), is also displayed (gray curve).
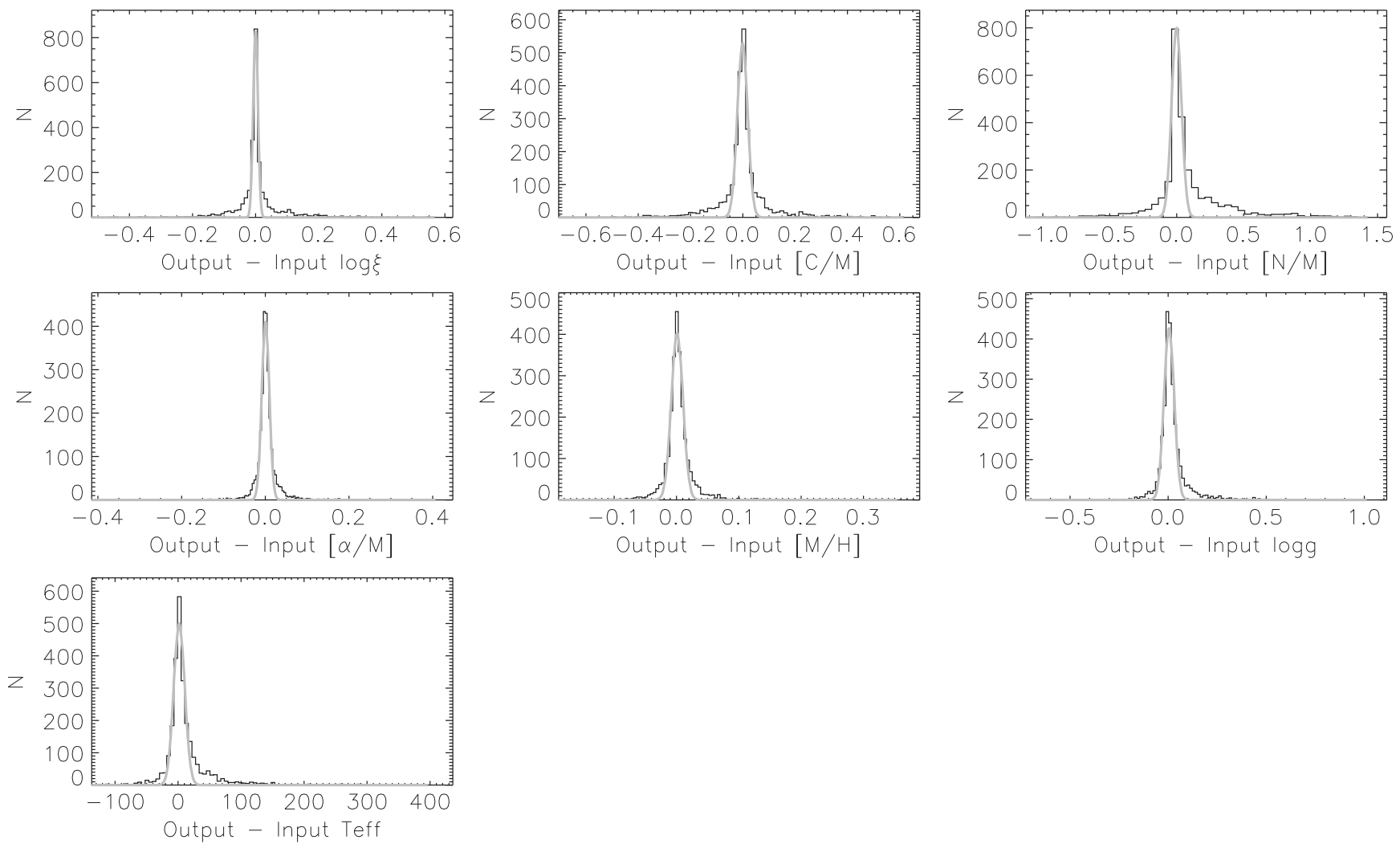

Figure 4. Same as Figure 3, but comparing both the input and the output from the ATLAS9/ASS $\epsilon$ T spectral library in the 7D parameter space. 
$\triangle \operatorname{logg}($ MARCS - ATLAS9) vs. $\triangle$ parameter (MARCS - ATLAS9) in low-gravity stars

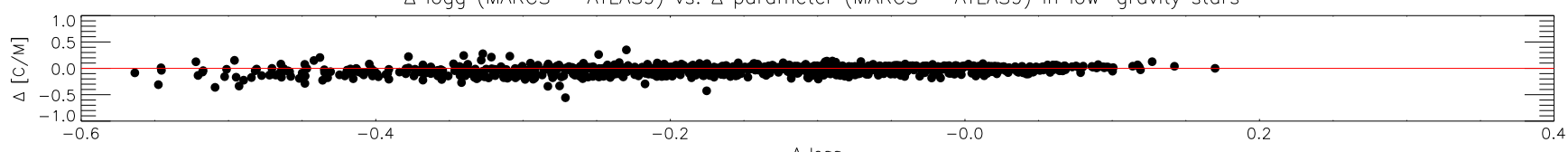

$\Delta \log 9$

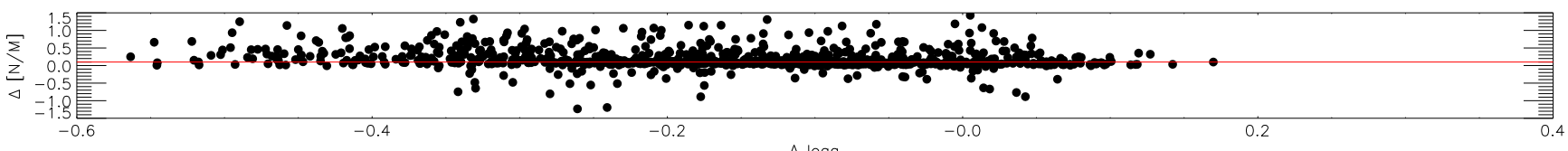

$\Delta \log g$
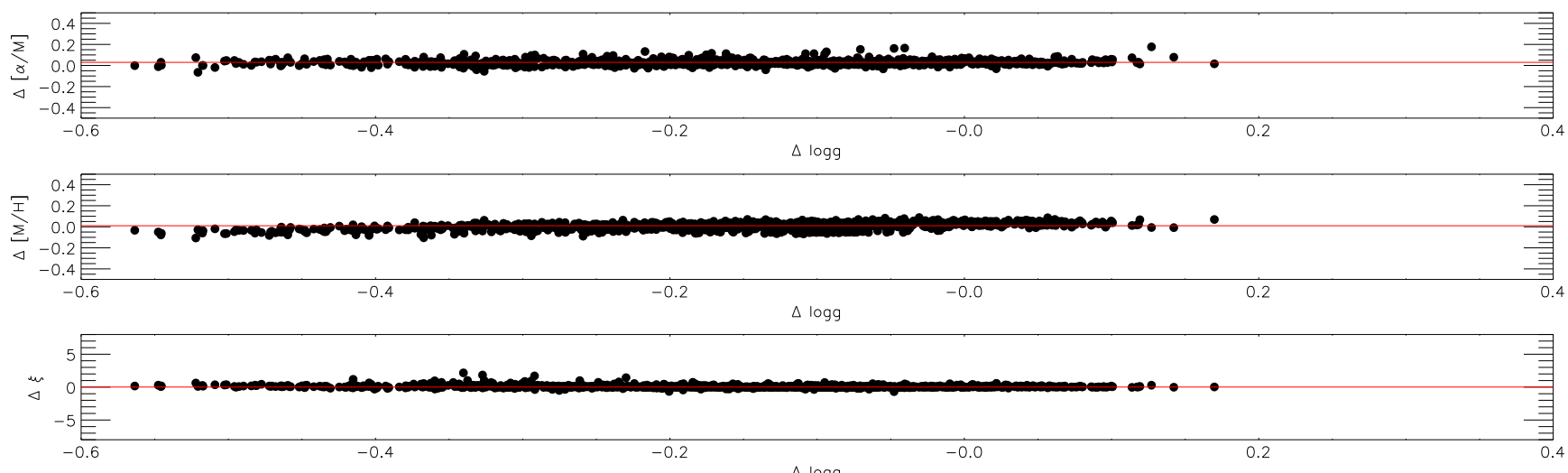

$\Delta \log 9$

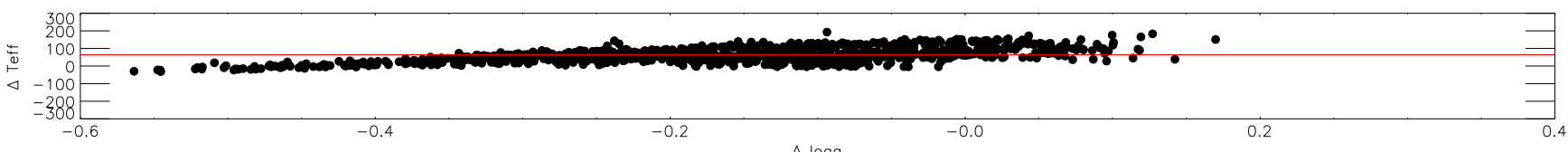

$\triangle \log g$

Figure 5. Parameter differences obtained between MARCS/Turbospectrum and ATLAS9/ASS $\epsilon$ T spectral libraries for low-gravity (log $g \leqslant 2.0)$ stars. From top to bottom: differences (MARCS-ATLAS9) in carbon $([\mathrm{C} / \mathrm{M}])$, nitrogen $([\mathrm{N} / \mathrm{M}])$, $\alpha$-elements $([\alpha / \mathrm{M}])$, metallicity $([\mathrm{M} / \mathrm{H}])$, microturbulent velocity $(\xi)$, and effective temperature $\left(T_{\text {eff }}\right)$ vs. MARCS-ATLAS9 differences in surface gravity $(\Delta \log g)$. The red line indicates the median values of the parameter differences.

results with the ones corresponding to the use of the ATLAS9/ ASS $\epsilon$ T library as input (observed spectra) but also running ASPCAP with the same ATLAS9/ASS $\epsilon$ T library. These results are reported in Table 2 (columns 4 and 5), where we a find good consistency for $\log g$ and $T_{\mathrm{eff}}$, which have median values of $0.01 \pm 0.04 \mathrm{dex}$ and $3.10 \pm 17.15 \mathrm{~K}$, respectively. We therefore conclude that the systematic differences between MARCS/ Turbospectrum and ATLAS9/ASS $\epsilon$ T for $\log g$ and $T_{\text {eff }}$ are significant. The nitrogen abundance $([\mathrm{N} / \mathrm{M}])$ may be slightly higher (median 0.09) and is the most problematic parameter to recover for the MARCS/Turbospectrum library, with the highest $\sigma$ of about 0.20 dex in $[\mathrm{N} / \mathrm{M}]$ (see Table 2). This $\mathrm{N}$ problem is not specific to the MARCS/Turbospectrum spectral library, since the [N/M] parameter displays also the highest $\sigma$ if we just compare the ATLAS9/ASS $\epsilon$ T synthetic spectra with themselves (see Table 2 and Figure 4). We note that ASPCAP is limited in accuracy for low-metallicity spectra $([\mathrm{M} / \mathrm{H}]<-1.0)$, since the scarcity of lines in that regime causes degeneracies among the stellar parameters (see García Pérez et al. 2015). Moreover, the $\sigma$ in $\mathrm{N}$ and in the other stellar parameters foundin tests by García Pérez et al. (2015) using ATLAS9/ASS $\epsilon$ T libraries are similar to the ones found here.

If we compare the MARCS-ATLAS9 residuals obtained for the 7D parameters by surface gravity groups, we find that highgravity stars $(\log g \geqslant 3.0)$ display higher $\sigma$ values (for all parameters) than the low-gravity stars ( $\log g \leqslant 2.0$; see Table 2 , columns 6-9). However, the median parameter values are quite similar with the exception of $T_{\mathrm{eff}}$, where the median value for low-gravity stars is significantly higher (by about $60 \mathrm{~K}$ ). The differences in the 7D parameters between the two $\log g$ groups become evident in Figures 5 and 6. These figures show the residuals in surface gravity $(\Delta \log g)$ versus the residuals in the other stellar parameters (from top to bottom: $[\mathrm{C} / \mathrm{M}],[\mathrm{N} / \mathrm{M}]$, $[\alpha / \mathrm{M}],[\mathrm{M} / \mathrm{H}], \xi$, and $T_{\text {eff }}$ ) for low-gravity stars (Figure 5) and high-gravity stars (Figure 6). For low-gravity stars (Figure 5), the residuals for most of the data points are close to their median values, with the exception of the already mentioned outliers (i.e., the largest and smallest $15.85 \%$ of the sample) and the $\mathrm{N}$ problem. These outliers are dominated by input ATLAS9/ASS $\epsilon$ T spectra of low-metallicity $([\mathrm{M} / \mathrm{H}]<-1.0 \mathrm{dex})$. In spite of the outliers, the results for the MARCS/Turbospectrum and ATLAS9/ASS $\epsilon$ T spectral libraries in low-gravity stars are very similar, and as mentioned above, the influence of possible sphericity effects on the derived abundances using ATLAS9 plane-parallel model atmospheres is small in the H-band ${ }^{31}$. High-gravity outliers in this analysis correspond mainly to the ATLAS9/ASS $\epsilon$ T input synthetic spectra with $[\mathrm{M} / \mathrm{H}]<-1.0$ dex. Further work by the APOGEE ASPCAP team is needed to fully understand why ASPCAP results degrate at high gravities.

\section{COMPARISONS OF THE SOLAR AND ARCTURUS SPECTRA WITH SYNTHESES FROM SPECTRAL LIBRARIES}

In order to further investigate how consistent the SDSS-III APOGEE spectral libraries are, we have fitted the Sun and

\footnotetext{
${ }^{31}$ Sphericity effects could be larger when comparing spherical and planeparallel model atmospheres with parameters $T_{\text {eff }}<3750 \mathrm{~K}$ and $\log \mathrm{g}<0.5$ (see, e.g., Hauschildt et al. 1999). These models were not included in the simulations performed in Section 3.
} 
$\triangle$ logg (MARCS - ATLAS9) vs. $\triangle$ parameter (MARCS - ATLAS9) in high-gravity stars
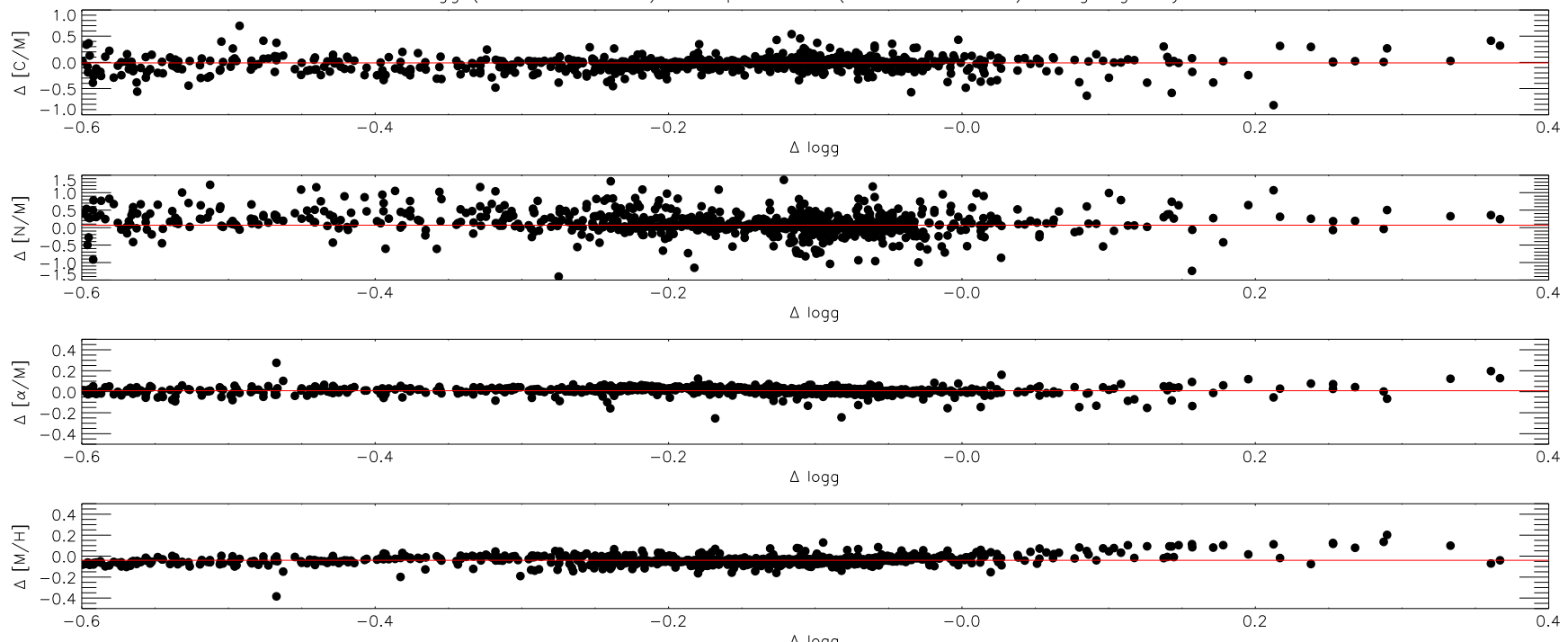

$\Delta \log 9$

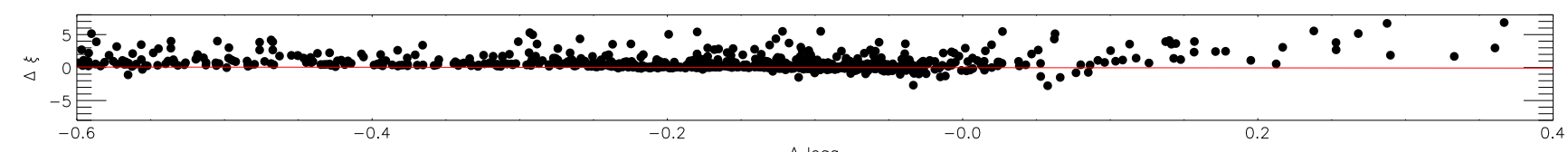

$\Delta \log 9$

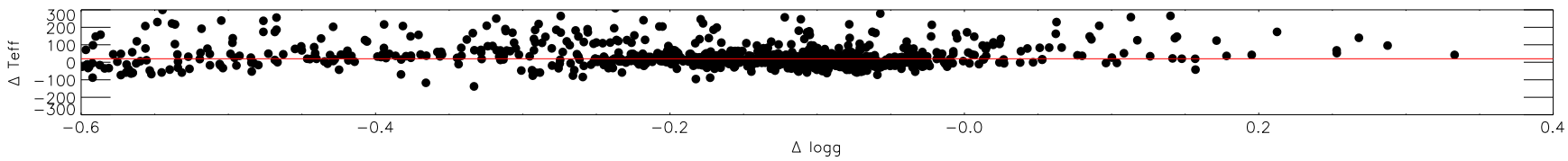

Figure 6. Same as Figure 5, but for high-gravity stars; i.e., $\log g \geqslant 3.0$.

Arcturus observed spectra using the two spectral libraries computed with both families of model atmospheres and spectral syntheses codes (ATLAS9/ASS $\epsilon$ T and MARCS/ Turbospectrum). We also compare with the synthetic spectra obtained by using ATLAS9 model atmospheres and the MOOG $^{32}$ spectral synthesis code (Sneden 1973). This is relevant because ATLAS9/MOOG synthetic spectra were used in the development of the DR12 line lists (see Shetrone et al. 2015). For the Sun, in particular, it is of interest to test how good our spectral libraries perform comparing with a spectrum at much higher spectral resolution than APOGEE's, and with well-known abundances. In addition, given that APOGEE observes mostly giant stars, we can verify how well our synthetic libraries reproduce the molecular lines (i.e., those suitable for $\mathrm{CNO}$ abundance determinations) using the spectrum of a cooler giant star as Arcturus.

For the Sun, we use the high-resolution flux spectrum $(R=400,000)$ by Livingston \& Wallace (1991). The Sun's synthetic spectra were computed adopting MARCS and ATLAS9 model atmospheres with $T_{\text {eff }}=5777 \mathrm{~K}$, $\log g=4.4370$, solar composition by Asplund et al. (2005), and a microturbulent velocity of $\xi=1.1 \mathrm{~km} \mathrm{~s}^{-1}$. Three different synthetic spectra were computed with the following model atmospheres and spectral synthesis codes: MARCS/Turbospectrum, ATLAS9/ASS $\epsilon$ T, and ATLAS9/MOOG. The solar macroturbulent velocity was taken into account in the synthetic spectra by convolving them with a Gaussian profile having a FWHM of $1.58 \mathrm{~km} \mathrm{~s}^{-1}$ (Allende Prieto et al. 2001). The synthetic spectra were also convolved with another Gaussian

\footnotetext{
32 http://www.as.utexas.edu/ chris/moog.html
}

profile to match the observed spectrum $(\mathrm{FWHM}=1.87 \mathrm{~km} \mathrm{~s}$ $\left.{ }^{-1}\right) \cdot{ }^{33}$ Finally, the spectra were interpolated to the wavelengths (in air) of the observed solar spectrum. All three computed syntheses were compared with the solar spectrum. The results from this comparison indicates a fairly good agreement between the observed synthetic spectra, as well as a good agreement between the synthetic spectra among themselves. The resulting global $\chi^{2}$ value for the Sun fitting were 15.05, 21.43 and 17.70 with the MARCS/Turbospectrum, ATLAS9/ ASS $\epsilon$ T, and ATLAS9/MOOG synthetic spectra, respectively. ${ }^{34}$ Figure 7 shows the quality of the fits to the solar spectrum for the spectral range 16500-16560 $\AA$, which includes several atomic and molecular lines. The differences (or residuals) between the different types of synthetic spectra and the Sun's observed spectrum are lower than $3 \%$ for most data points. The rms value for the MARCS/Turbospectrum synthetic spectrum is slightly lower $(\sim 0.1 \%)$ than the ones for the ATLAS9/ ASS $\epsilon$ T and ATLAS9/MOOG spectra. We note, however, that a perfect match between synthetic and observed spectra is not expected because of the convective line shifts and asymmetries in the real stars, and of course because our modeling of the solar atmosphere is not perfect.

In the case of the giant star Arcturus, we have used the Fourier transform spectrometer (FTS) observed spectrum smoothed to the resolution of APOGEE $(R=22,500)$ and the best fit

\footnotetext{
33 The macroturbulence of $1.58 \mathrm{~km} \mathrm{~s}^{-1}$ is from optical spectra (Allende Prieto et al. 2001) and we need an extra macroturbulence contribution to match the observed H-band solar spectrum.

${ }^{34}$ We estimate the error on the observed spectrum calculating the standard deviation over a spectral region free of absorption lines and assuming this error constant along the full range in wavelength.
} 


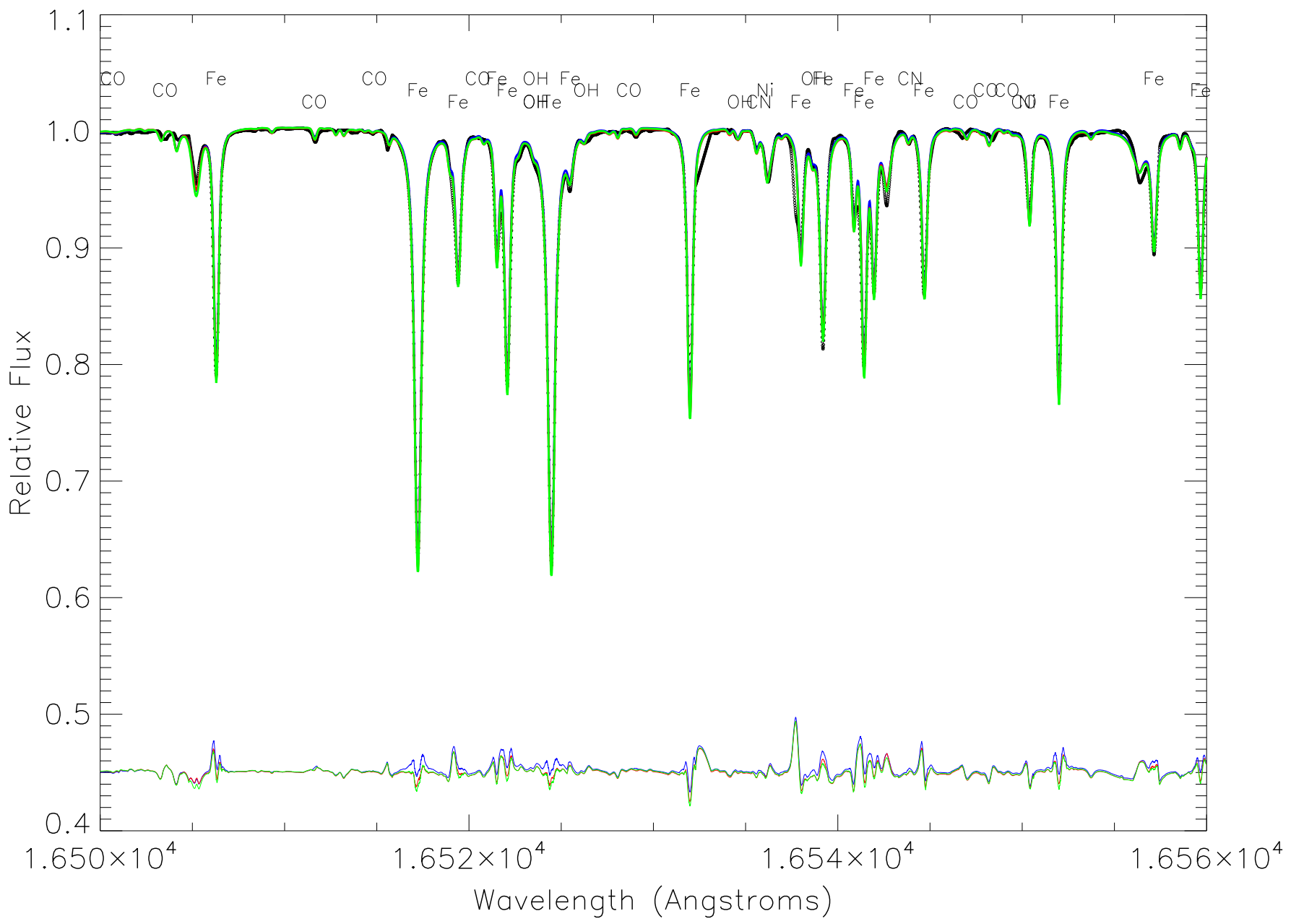

Figure 7. High-resolution H-band observed spectrum of the Sun (black dots) in the 16500-16560 ̊̊ region vs. the best fits obtained using MARCS/Turbospectrum (red line), ATLAS9/ASS $\epsilon$ T (blue line), and ATLAS9/MOOG (green line) synthetic spectra. All spectra are expressed in air wavelengths. The residuals, computed as flux (synthetic-observed) +0.45 , are plotted at the bottom with the same colors. The spectral features identified by Hinkle et al. (1995) are indicated at the top.

spectrum from each library provided by ASPCAP/FERRE ${ }^{35}$ (see also next section for further details). The reduced global $\log \chi^{2}$ value for the fitting was 0.40 for the MARCS/Turbospectrum library and 0.41 for the ATLAS9/ASS $\epsilon$ T one (see Table 3 and Section 5). We focus our comparisons of molecular features in those spectral regions selected in the line-by-line abundance analysis by Smith et al. (2013). These authors used four windows to extract the ${ }^{12} \mathrm{C}$ abundance from ${ }^{12} \mathrm{C}^{16} \mathrm{O}$ lines (15578-15586, 15774-15787, 15976-16000, and 16183-16196 ^), four windows to extract the ${ }^{16} \mathrm{O}$ abundance from ${ }^{16} \mathrm{OH}$ lines (15277-15282, 15390-15392, 15504-15507, and 16189-16193
$\AA)$, and nine molecular lines of ${ }^{12} \mathrm{C}^{14} \mathrm{~N}(15260,15322,15397$, $15332,15410,15447,15466,15472$, and $15482 \AA)$ to extract the abundance of ${ }^{14} \mathrm{~N}$.

The average of the residuals to fits to the observed Arcturus spectrum were derived independently in each region/line for the two synthetic libraries. Very small (rms $\sim 0.1 \%-0.3 \%$ ) differences are found between syntheses from both libraries and the observed spectrum (and between the library synthetic spectra themselves). Figures 8 and 9 display the fits in the ${ }^{12} \mathrm{C}^{16} \mathrm{O}$ and ${ }^{16} \mathrm{OH}$ spectral regions and, in the ${ }^{12} \mathrm{C}^{14} \mathrm{~N}$ spectral lines mentioned above. In the ${ }^{12} \mathrm{C}^{16} \mathrm{O}$ windows, the MARCS/

Table 3

ATLAS9/ASS $\epsilon$ T vs. MARCS/Turbospectrum $\log \chi^{2}$ Values in FTS Stars

\begin{tabular}{lcr}
\hline \hline Star & $\log \chi^{2}$ ATLAS9/ASS $\epsilon$ T & $\log \chi^{2}$ MARCS/Turbospectrum \\
\hline$\alpha$ Boo (FTS) & 0.4075 & 0.3972 \\
$\alpha$ Boo (atlas) & 0.3524 & 0.3443 \\
$\alpha$ Boo (NMSU 1 m) & 2.0242 & 2.0236 \\
$\mu$ Leo (FTS) & 1.1852 & 1.1885 \\
$\mu$ Leo (NMSU 1 m) & 1.8115 & 1.8260 \\
$\beta$ And (FTS) & 1.3926 \\
$\delta$ Oph (FTS) & 1.3842 & 1.1730 \\
\hline
\end{tabular}

\footnotetext{
${ }^{a}$ These stars have atmospheric parameters corresponding to a hole in the MARCS/Turbospectrum grid.
} 

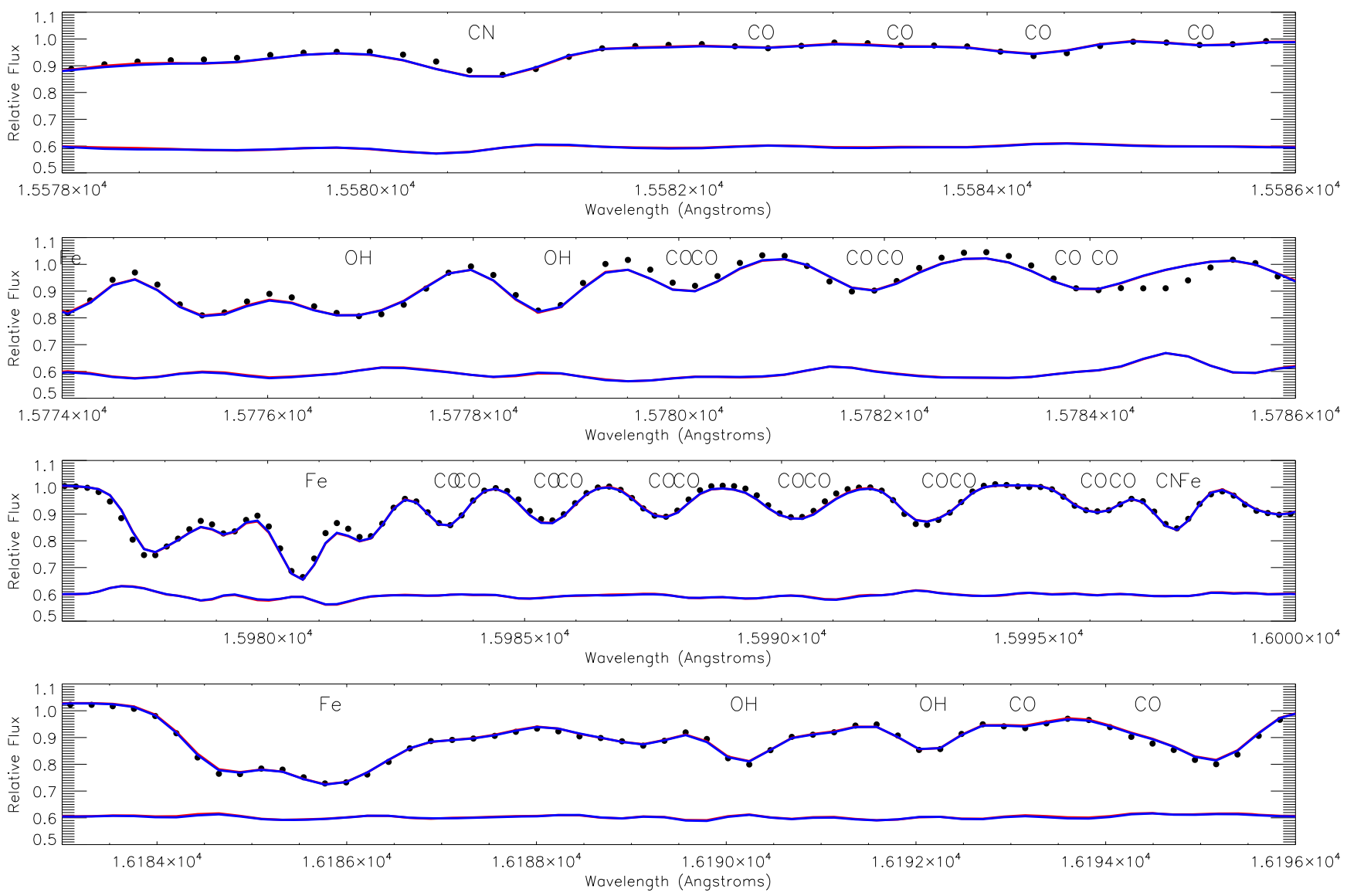

Figure 8. Arcturus FTS observed spectrum (black dots) smoothed to the APOGEE resolution $(R=22,500)$, showing four ${ }^{12} \mathrm{C}^{16} \mathrm{O}$ molecular windows/regions. The best fits obtained with the MARCS/Turbospectrum (red line) and ATLAS9/ASS $\epsilon$ T (blue line) synthetic spectra are also shown. All spectra are expressed in air wavelengths. The residuals, computed as flux(synthetic-observed) +0.60 , are plotted at the bottom with the same color code. The spectral features identified by Hinkle et al. (1995) are indicated at the top.

Turbospectrum library spectrum fits slightly better the regions at 15578-15586 and 15976-16000 , while the ATLAS9/ ASS $\epsilon$ T library spectrum fits better the regions at 15774-15787 and 16183-16196 $\AA$. For the ${ }^{16} \mathrm{OH}$ windows, the MARCS/ Turbospectrum library spectrum fits better the regions at 15277-15282 and 15390-15392 A, while the ATLAS9/ASS $\epsilon$ T library spectrum fits better the regions at 15504-15507 and 16189-16193 $\AA$. Finally, for the ${ }^{12} \mathrm{C}^{14} \mathrm{~N}$ lines, the MARCS/ Turbospectrum library spectrum fits slightly better the lines at 15260, 15397, 15466, and 15482 $\AA$, while the ATLAS9/ ASS $\epsilon$ T library spectrum fits better the lines at 15322, 15332, 15447 , and $15472 \AA$. Both libraries provide just the same residual average for the $\mathrm{CN}$ line at $15410 \AA$. We conclude that both synthetic spectral libraries provide an excellent fit to the molecular features in the spectrum of Arcturus; the rms values between both synthetic libraries are no significant and of the order of only $\sim 0.1 \%-0.3 \%$. $^{36}$

\section{APPLICATION OF THE APOGEE SPECTRAL LIBRARIES TO SELECTED GIANT STARS}

Given that most of the APOGEE sample are red giant stars, we are interested here in exploring the abundance differences, as obtained by the APOGEE spectral libraries, in a small sample of well known giant stars observed at very high

\footnotetext{
35 FERRE is available from http://hebe.as.utexas.edu/ferre.

36 Note that the f-values for atomic lines were tuned to match the Sun and Arcturus but not those of molecular transitions (see Shetrone et al. 2015).
}

resolution. Smith et al. (2013) derived chemical abundances from a line-by-line analysis of 15 elements in several wellknown bright field giants and explored what elements can be analyzed from APOGEE spectra. The sample analyzed here consists in the four Smith et al. (2013) stars with $T_{\mathrm{eff}}>3500$ $\mathrm{K}$; this includes two M-giants ( $\beta$ And and $\delta$ Oph) and two Kgiants ( $\alpha$ Boo and $\mu$ Leo). For their study, Smith et al. used high-resolution spectra in the H-band acquired with the FTS (Hall et al. 1979) installed at the Coude focus of the Kitt Peak National Observatory $4 \mathrm{~m}$ Mayall reflector. The spectral resolution of these FTS spectra varies from 45,000 to 100,000 . The original spectra cover a wavelength range larger than that of APOGEE, but Smith et al. (2013) restricted their analysis to the spectral range from 1500 to $1700 \mathrm{~nm}$. The infrared atlas spectrum of $\alpha$ Boo (Arcturus) by Hinkle et al. (1995), obtained with the same instrument at a resolution of 100,000 , is added to our sample of starswith FTS spectra. The data were smoothed to the APOGEE resolution (i.e., $R=22,500)$ by convolving with a Gaussian kernel. We also converted the resulting convolved spectra to the APOGEE apStar FITS format described by Holtzman et al. (2015). Our FTS sample includes the H-band spectra of $\alpha$ Boo and $\mu$ Leo obtained with the APOGEE spectrograph, but using the New Mexico State University 1.0-meter Telescope (Holtzman et al. 2015; NMSU $1 \mathrm{~m}$, hereafter). The NMSU $1 \mathrm{~m}$ spectra are reduced with the APOGEE data reduction pipeline (Nidever et al. 2015). All spectra mentioned above were processed with a quick version of ASPCAP, QASPCAP, which 

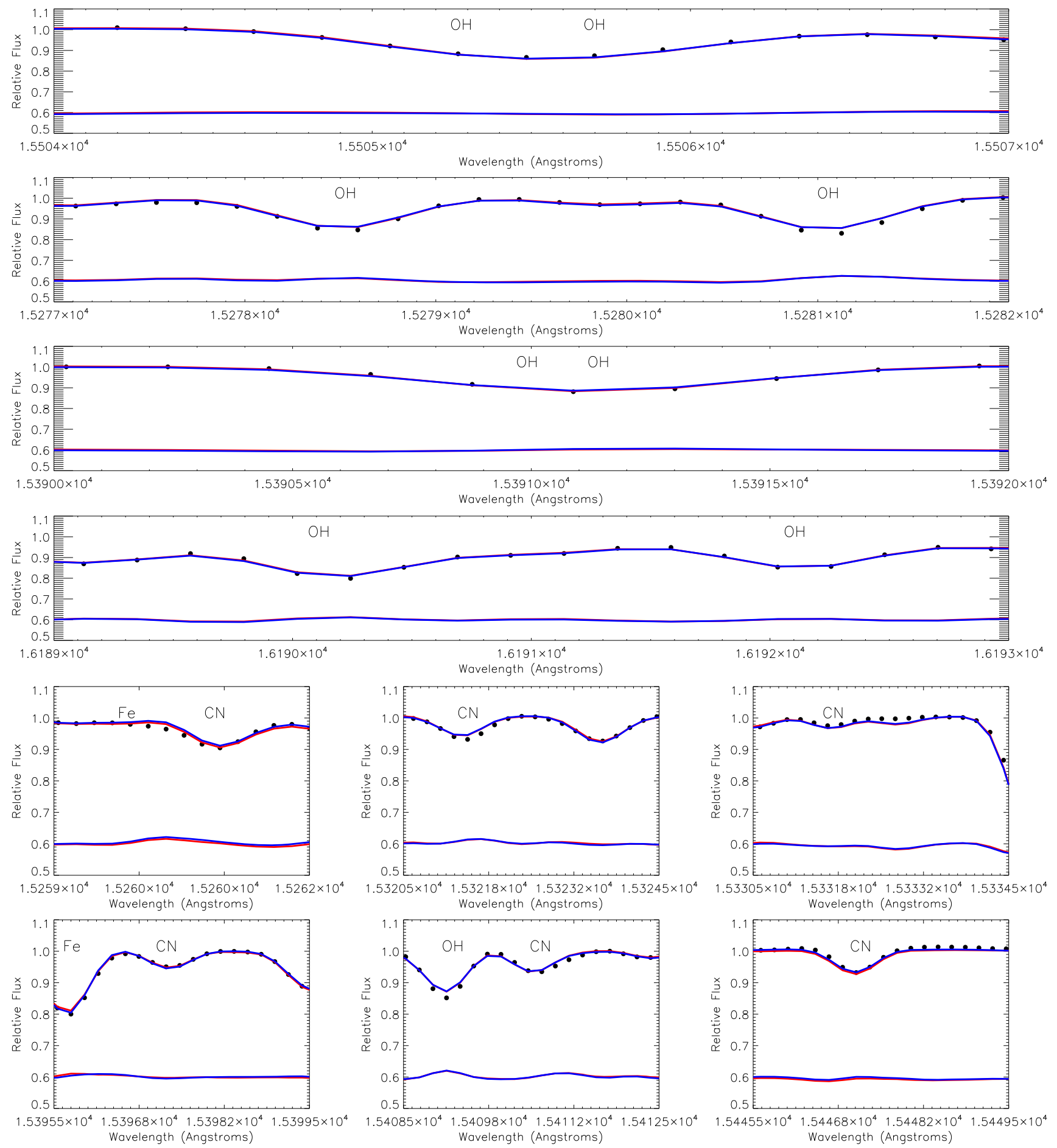

$1.53955 \times 10^{4} \quad 1.53968 \times 10^{4} \quad 1.53982 \times 10^{4} \quad 1.53995 \times 10^{4}$ Wavelength (Angstroms)
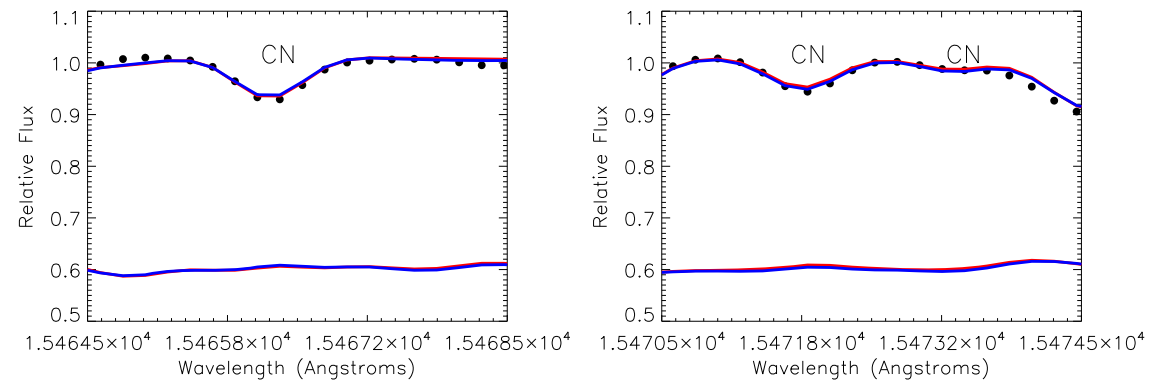

$1.54455 \times 10^{4} \quad 1.54468 \times 10^{4} \quad 1.54482 \times 10^{4} \quad 1.54495 \times 10^{4}$

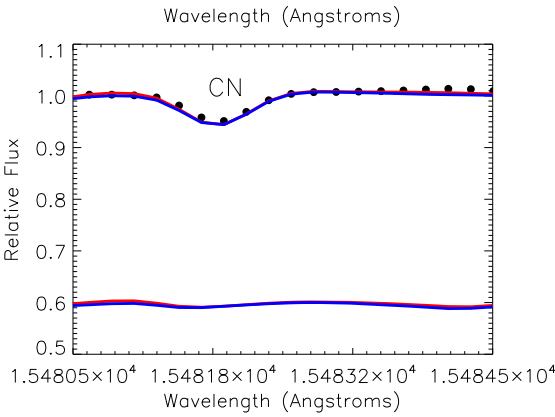

Figure 9. Arcturus FTS observed spectrum (black dots) smoothed to the APOGEE resolution $(R=22,500)$ and the synthetic spectra in four ${ }^{16} \mathrm{OH}$ molecular windows/regions (upper panel) and in nine ${ }^{12} \mathrm{C}^{14} \mathrm{~N}$ lines (lower panel). Symbols and colors as in Figure 8. The spectral features identified by Hinkle et al. (1995) are indicated at the top. 
Table 4

ATLAS9/ASS $\epsilon$ T vs. MARCS/Turbospectrum Stellar Parameters in FTS Stars

\begin{tabular}{|c|c|c|c|c|c|c|c|c|}
\hline \multirow[b]{2}{*}{ Star } & \multicolumn{3}{|c|}{ ATLAS9/ASS $\epsilon$ T } & \multicolumn{5}{|c|}{ MARCS/Turbospectrum } \\
\hline & $T_{\text {eff }}$ & $\log g$ & {$[\mathrm{M} / \mathrm{H}]$} & $\xi$ & $T_{\text {eff }}$ & $\log g$ & {$[\mathrm{M} / \mathrm{H}]$} & $\xi$ \\
\hline$\alpha$ Boo (FTS) & 4187 & 2.04 & -0.40 & 2.03 & 4192 & 1.85 & -0.47 & 2.07 \\
\hline$\alpha$ Boo (atlas) & 4188 & 2.07 & -0.43 & 1.90 & 4192 & 1.95 & -0.48 & 1.92 \\
\hline$\alpha$ Boo (NMSU $1 \mathrm{~m}$ ) & 4208 & 2.07 & -0.50 & 1.15 & 4223 & 1.92 & -0.56 & 1.25 \\
\hline$\mu$ Leo (FTS) & 4493 & 2.80 & 0.44 & 1.93 & 4520 & 2.76 & 0.40 & 1.99 \\
\hline$\mu$ Leo (NMSU $1 \mathrm{~m})$ & 4560 & 2.98 & 0.36 & 1.00 & 4551 & 2.87 & 0.30 & 1.04 \\
\hline$\beta$ And $(\mathrm{FTS})^{\mathrm{a}}$ & 3823 & 1.16 & -0.20 & 2.36 & 3791 & 1.19 & -0.24 & 2.42 \\
\hline$\delta$ Oph $(\mathrm{FTS})^{\mathrm{a}}$ & 3832 & 1.45 & 0.03 & 2.21 & 3809 & 1.48 & -0.04 & 2.31 \\
\hline
\end{tabular}

a These stars have atmospheric parameters corresponding to a hole in the MARCS/Turbospectrum grid.

is short version of ASPCAP, and prepares the spectra for the automated fitting with FERRE (see García Pérez et al. 2015 for a detailed description of ASPCAP). Finally, we derived the atmospheric parameters and chemical abundances of 15 elements (see below) with FERRE, interpolating in the ATLAS9/ASS $\epsilon$ T and MARCS/Turbospectrum synthetic grids described in this paper.

The atmospheric parameters and chemical abundances obtained are listed in Tables 4 and 5, respectively. For all stars, we find a very good agreement between the values obtained with both synthetic grids. The $\log \chi^{2}$ of the fits for each spectral library are quite similar (Table 3 ). The differences of the derived $T_{\text {eff }}, \log g$, microturbulent velocity (in log scale), and $[\mathrm{M} / \mathrm{H}]$ for each star (and synthetic grid) are plotted in Figure 10. The atmospheric parameters derived here for the Arcturus giant are somewhat different from those adopted in Smith et al. (2013): $\Delta T_{\text {eff }}<90 \mathrm{~K}, \Delta \log g<0.4$ dex, $\Delta \xi<0.6$ $\mathrm{km} \mathrm{s}^{-1}, \Delta[\mathrm{M} / \mathrm{H}]<0.2$ dex. In Smith et al. (2013) the effective temperatures were based on the $(\mathrm{J}-\mathrm{K})$ color and derived from an average of two calibrations: González Hernández \& Bonifacio (2009) and Bessell et al. (1998). Their surface gravities were obtained from evolutionary tracks and microturbulent velocities from the $\mathrm{Fe}$ I lines. The stellar parameters derived here are purely spectroscopic. In addition, it is important to note that Smith et al. (2013) carried out a lineby-line chemical abundance analysis using the MOOG synthesis code and a so-called intermediate version of the APOGEE line list (line list INT; Shetrone et al. 2015) that is previous to the DR12 APOGEE line list used here.

Regarding abundances, we find in general very good agreement $(<0.1 \mathrm{dex})$ between the chemical abundances obtained by the ATLAS9/ASS $\epsilon$ T and MARCS/Turbospectrum stellar spectral libraries in the FTS stars (see Figure 11). For Arcturus ( $\alpha$ Boo), MARCS/Turbospectrum performs slightly better than ATLAS9/ASS $\epsilon$ T, with the exception of $\mathrm{N}$ (see below). Despite the higher MARCS/Turbospectrum $T_{\text {eff }}$ values, the set of derived MARCS/Turbospectrum abundances in Arcturus (and also in $\mu$ Leo) are slightly lower $(<0.1 \mathrm{dex})$ than those from ATLAS9/ASS $\epsilon$ T because of the generally lower metallicity and gravity obtained (which compensates the expected abundance increase due to a higher $T_{\text {eff }}$; Section 3) with the MARCS/Turbospectrum library (see Tables 4 and 5). However, $\mathrm{N}$ seems to be more affected and the MARCS/ Turbospectrum derived $\mathrm{N}$ abundances can be lower by 0.15 dex. On the other hand, ATLAS9/ASS $\epsilon$ T fits slightly better than MARCS/Turbospectrum in the two FTS stars $\beta$ And and $\delta$ Oph; especially concerning nitrogen, where MARCS/Turbospectrum gives $\mathrm{N}$ abundances lower by $\sim 0.2-0.3$ dex (see Figure 11 ). This, however, is likely due to the use of the cooler $3700 \mathrm{~K}$ MARCS model in place of the $3750 \mathrm{~K}$ one, which is lacking in the MARCS grid, to interpolate their exact matching value of effective temperatures of about $3825-3850 \mathrm{~K}$.

In short, based on the results from the comparisons performed in this study, there is good indication that the MARCS/Turbospectrum library, although with several more models missing from the grid, gives results comparable to those from the ATLAS9/ASS $\epsilon$ T library. ${ }^{37}$

\section{CONCLUSIONS AND FUTURE WORK}

We present the stellar spectral libraries for the final data release of the SDSS-III APOGEE survey, which are used for the automated chemical analysis of survey data. The spectral libraries employed in the data release 12 (DR12; Alam et al. 2015) are constructed for a wide range in effective temperature $\left(T_{\text {eff }}\right.$ ranging from 3500 to $\left.8000 \mathrm{~K}\right)$ and are based on ATLAS9 model atmospheres and the ASS $\epsilon$ T spectral synthesis code. We also present here a second family of SDSSIII APOGEE stellar spectral libraries based on MARCS model atmospheres and the Turbospectrum spectral synthesis code. The ATLAS9/ASS $\epsilon$ T $\left(T_{\text {eff }}=3500-8000 \mathrm{~K}\right)$ and MARCS/ Turbospectrum $\left(T_{\text {eff }}=3500-5500 \mathrm{~K}\right)$ synthetic grids have seven dimensions (7D), covering a wide metallicity $([\mathrm{M} / \mathrm{H}])$, surface gravity $(\log g)$, microturbulence $(\xi)$, carbon $([\mathrm{C} / \mathrm{M}])$, nitrogen $([\mathrm{N} / \mathrm{M}])$, and $\alpha$-element $([\alpha / \mathrm{M}])$ ranges of variation.

We have compared both ATLAS9/ASS $\epsilon$ T and MARCS/ Turbospectrum spectral libraries to a test sample of 2552 synthetic spectra with the same 7D stellar parameters. The differences found between both families of synthetic spectra are very small ( $\mathrm{rms}$ values of the order of only $\sim 0.3 \%-0.6 \%$ ). Interestingly, we find that the sphericity effects in the H-band seem to be smaller than those previously found in the optical range and the ASSeT and Turbospectrum spectral synthesis codes provide very similar synthetic spectra. By fitting the GKATLAS9/ASS $\epsilon$ T library with the K-MARCS/Turbospectrum library, we have found small systematic differences in the seven main stellar parameters $\left(7 \mathrm{D} ; T_{\text {eff }},[\mathrm{M} / \mathrm{H}], \log g, \xi,[\mathrm{C} / \mathrm{M}]\right.$, $[\mathrm{N} / \mathrm{M}]$, and $[\alpha / \mathrm{M}])$ automatically provided by the SDSS-III APOGEE survey for low-gravity stars $(\log g \leqslant 2.0)$. The outliers correspond to low-metallicity $([\mathrm{M} / \mathrm{H}]<-1.0)$ synthetic spectra. However, the results for high-gravity $(\log g>3.0)$

\footnotetext{
${ }^{37}$ García Pérez et al. (2015) used the same ATLAS9/ASS $\epsilon$ T spectral library to analyze the spectra of FTS stars but with a different order in the stellar parameters.
} 
Table 5

ATLAS9/ASS $\epsilon$ T vs. MARCS/Turbospectrum Element Abundances in FTS Stars

\begin{tabular}{|c|c|c|c|c|c|c|c|c|c|c|c|c|c|}
\hline Star & {$[\mathrm{Fe} / \mathrm{H}]$} & {$[\mathrm{C} / \mathrm{H}]$} & {$[\mathrm{N} / \mathrm{H}]$} & {$[\mathrm{O} / \mathrm{H}]$} & {$[\mathrm{Mg} / \mathrm{H}]$} & {$[\mathrm{Al} / \mathrm{H}]$} & {$[\mathrm{Si} / \mathrm{H}]$} & {$[\mathrm{K} / \mathrm{H}]$} & {$[\mathrm{Ca} / \mathrm{H}]$} & {$[\mathrm{Ti} / \mathrm{H}]$} & {$[\mathrm{V} / \mathrm{H}]$} & {$[\mathrm{Mn} / \mathrm{H}]$} & {$[\mathrm{Ni} / \mathrm{H}]$} \\
\hline & \multicolumn{13}{|c|}{ ATLAS9/ASS $\epsilon$ T } \\
\hline$\alpha$ Boo (FTS) & -0.42 & -0.29 & -0.30 & -0.16 & -0.23 & -0.24 & 0.01 & -0.44 & -0.37 & -0.43 & -0.53 & -0.48 & -0.38 \\
\hline$\alpha$ Boo (atlas) & -0.45 & -0.31 & -0.35 & -0.20 & -0.25 & -0.37 & -0.10 & -0.45 & -0.42 & -0.43 & -0.58 & -0.49 & -0.39 \\
\hline$\alpha$ Boo (NMSU $1 \mathrm{~m}$ ) & -0.52 & -0.46 & -0.58 & -0.39 & -0.39 & -0.27 & -0.30 & -0.51 & -0.51 & -0.26 & -0.71 & -0.55 & -0.32 \\
\hline$\mu$ Leo (FTS) & 0.44 & 0.43 & 0.91 & 0.49 & 0.36 & 0.50 & 0.53 & 0.50 & 0.23 & 0.62 & 0.30 & 0.50 & 0.50 \\
\hline$\mu$ Leo (NMSU $1 \mathrm{~m}$ ) & 0.31 & 0.37 & 0.73 & 0.37 & 0.22 & 0.41 & 0.33 & 0.19 & 0.21 & 0.64 & 0.32 & 0.50 & 0.47 \\
\hline$\beta$ And (FTS) & -0.21 & -0.35 & 0.20 & -0.11 & -0.07 & -0.12 & -0.06 & -0.30 & -0.26 & -0.07 & -0.23 & -0.11 & -0.19 \\
\hline \multirow[t]{2}{*}{$\delta$ Oph (FTS) } & 0.00 & -0.08 & 0.30 & 0.08 & 0.06 & 0.19 & 0.24 & 0.27 & -0.08 & 0.17 & -0.04 & 0.21 & 0.03 \\
\hline & \multicolumn{13}{|c|}{ MARCS/Turbospectrum } \\
\hline$\alpha$ Boo (FTS) & -0.49 & -0.37 & -0.38 & -0.17 & -0.26 & -0.24 & -0.06 & -0.45 & -0.37 & -0.45 & -0.56 & -0.53 & -0.43 \\
\hline$\alpha$ Boo (atlas) & -0.51 & -0.36 & -0.50 & -0.22 & -0.28 & -0.37 & -0.15 & -0.47 & -0.42 & -0.43 & -0.59 & -0.53 & -0.42 \\
\hline$\alpha$ Boo (NMSU $1 \mathrm{~m}$ ) & -0.59 & -0.50 & -0.64 & -0.39 & -0.40 & -0.26 & -0.36 & -0.52 & -0.51 & -0.35 & -0.72 & -0.58 & -0.37 \\
\hline$\mu$ Leo (FTS) & 0.38 & 0.40 & 0.91 & 0.47 & 0.32 & 0.50 & 0.41 & 0.50 & 0.23 & 0.58 & 0.30 & 0.50 & 0.50 \\
\hline$\mu$ Leo (NMSU $1 \mathrm{~m})$ & 0.24 & 0.31 & 0.73 & 0.32 & 0.20 & 0.41 & 0.27 & 0.15 & 0.18 & 0.57 & 0.26 & 0.50 & 0.41 \\
\hline$\beta$ And $(\text { FTS })^{\mathrm{a}}$ & -0.27 & -0.44 & -0.08 & -0.34 & -0.03 & -0.29 & -0.02 & -0.36 & -0.38 & -0.28 & -0.36 & -0.15 & -0.23 \\
\hline$\delta \mathrm{Oph}(\mathrm{FTS})^{\mathrm{a}}$ & -0.07 & -0.16 & 0.07 & -0.11 & -0.11 & -0.02 & 0.27 & 0.19 & -0.19 & -0.08 & -0.15 & 0.17 & -0.02 \\
\hline
\end{tabular}

${ }^{a}$ These stars have atmospheric parameters corresponding to a hole in the MARCS/Turbospectrum grid. 

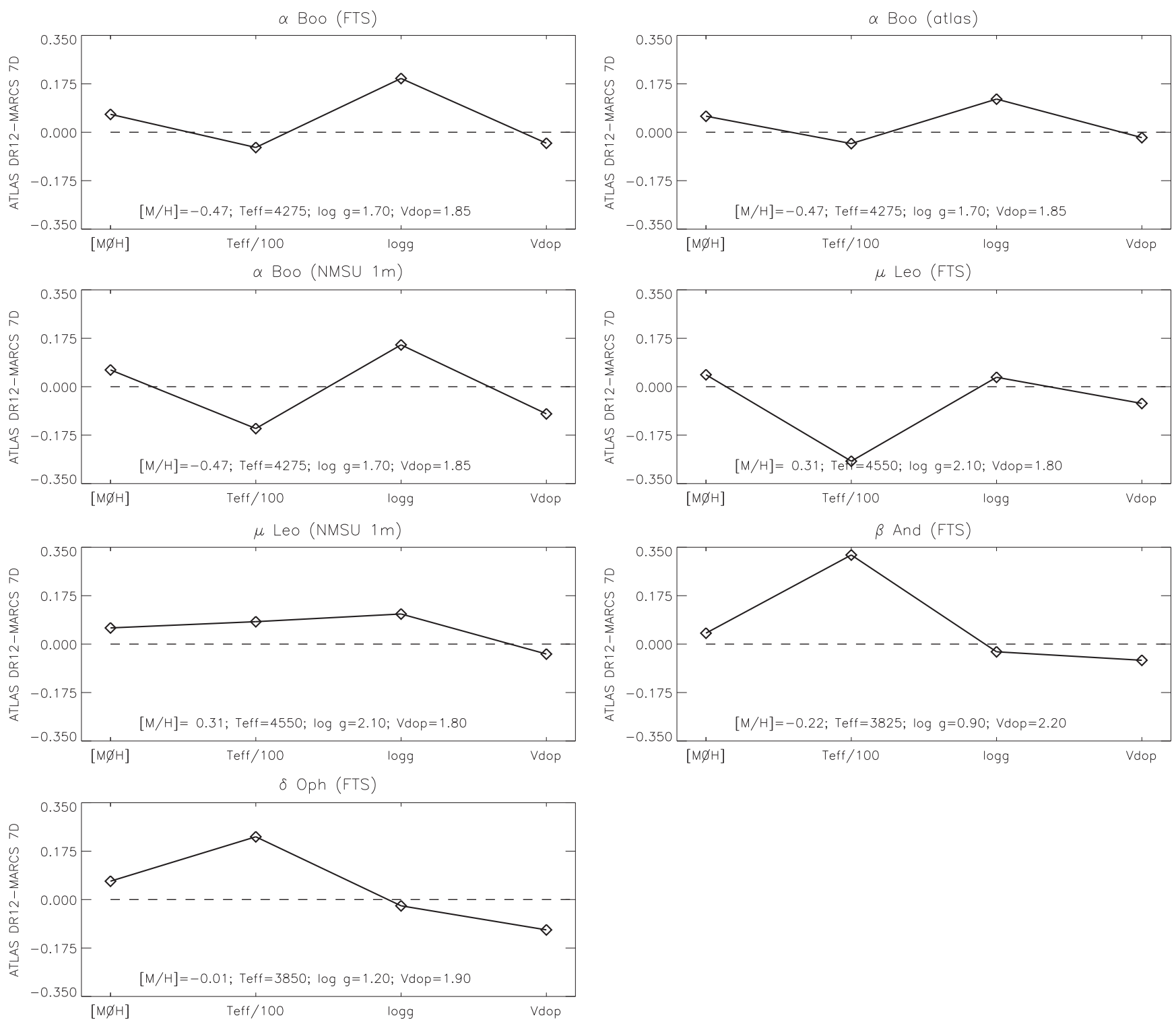

Figure 10. Comparison between the atmospheric parameters derived by FERRE using ATLAS9/ASS $\epsilon$ T and MARCS/Turbospectrum for the calibration sample observed with the FTS. In order to have all the values in the same scale, the $T_{\text {eff }}$ value has been divided by 100 . Moreover, the microturbulent velocity $\left(\xi \equiv v_{\text {dop }}\right)$ is in logarithmic scale, while the dashed line is the zero point. Note that stars $\beta$ And and $\delta$ Oph have atmospheric parameters that correspond to a hole in the MARCS/ Turbospectrum grid (see the text).

stars are worse than the former ones, and the average scatter for the entire parameter space is higher than in low-gravity stars. These outliers are also dominated by synthetic spectra with $[\mathrm{M} / \mathrm{H}]<-1.0$ dex. Further work by the APOGEE ASPCAP team is needed to completely understand the presence of these outliers in the 7D parameter space.

Both the DR12 SDSS-III APOGEE synthetic spectral library as well as the additional spectral library based on the MARCS model atmospheres provide almost identical model fits to the observed spectra of the Sun, Arcturus, and the stars with FTS spectra. For example, they give an excellent fit to the Sun's spectrum as well as to the molecular features $(\mathrm{CO}, \mathrm{OH}$, and $\mathrm{CN}$ ) in the spectrum of Arcturus; the differences (or residuals) between these synthetic libraries are of the order of only $\sim 0.1 \%-0.3 \%$ (rms). We conclude that both SDSS-III APOGEE synthetic spectral libraries provide very similar results (i.e., atmospheric parameters and chemical abundances), which supports the use of the ATLAS9/ASS $\epsilon$ T synthetic grids (which otherwise cover a parameter space much wider than the actual MARCS/Turbospectrum grid) in DR12. The SDSS-III APOGEE synthetic spectral libraries presented here are publicly available online and they can be used also for chemical analysis in the H-band making use of other available high-resolution spectroscopic instruments working in the H-band.

The APOGEE stellar spectral libraries presented here will be improved for the SDSS-IV/APOGEE-2 survey, and periodically updated in the future. We plan to extend the MARCS/ Turbospectrum stellar spectral library to cooler temperatures $\left(2500 \leqslant T_{\text {eff }} \leqslant 3500 \mathrm{~K}\right)$. The effect of other molecules such as $\mathrm{H}_{2} \mathrm{O}$ and $\mathrm{FeH}$ may be important at these extremely cool effective temperatures and we will need to update the present APOGEE linelist by including these molecules. Finally, we plan to evaluate the effects of the missing opacities for polyatomic molecules (like $\mathrm{HCN}, \mathrm{C}_{2} \mathrm{H}_{2}$ ) on the structures of 

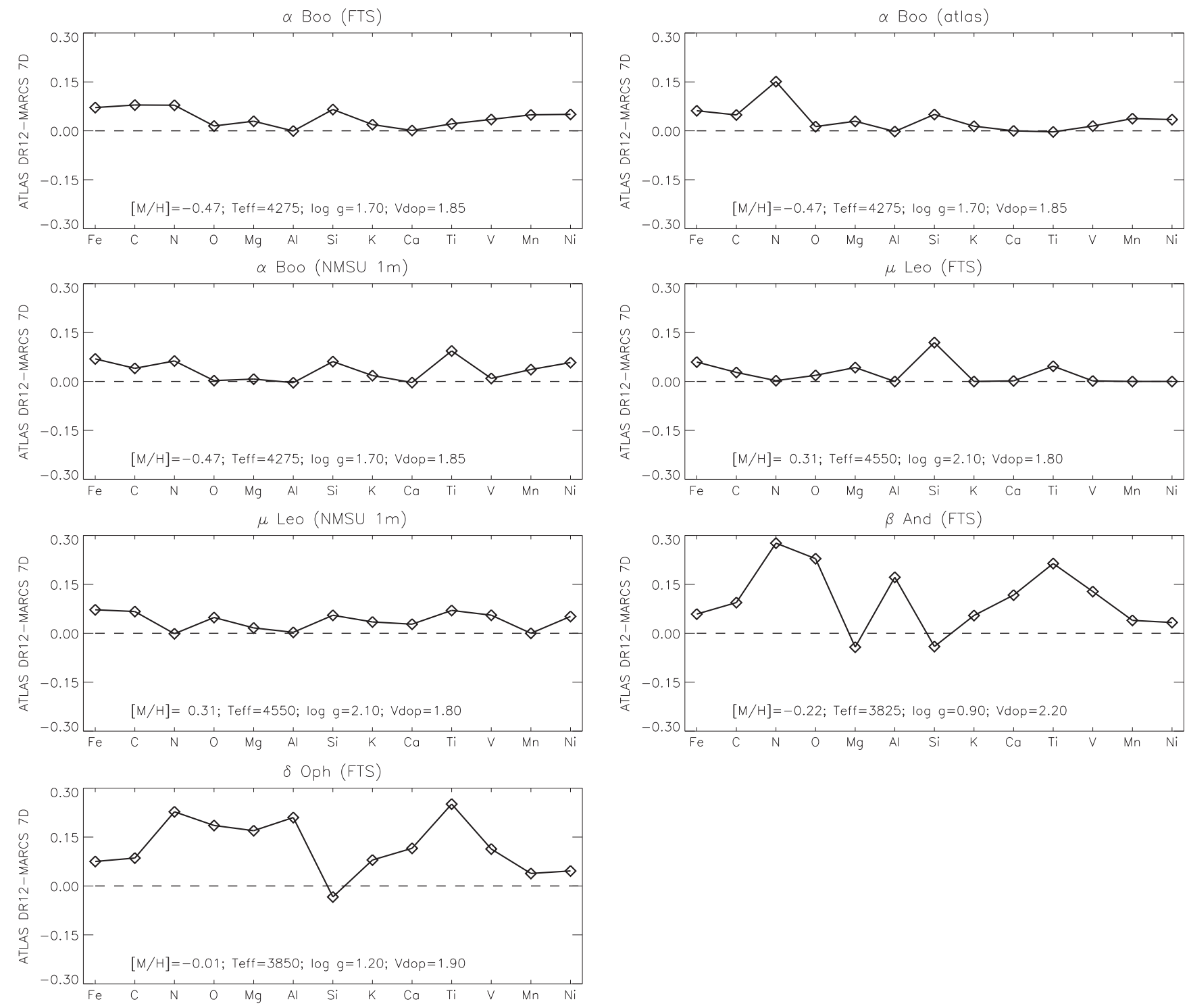

Figure 11. Same as Figure 10, but for the abundances of individual elements. Note that stars $\beta$ And and $\delta$ Oph have atmospheric parameters that correspond to a hole in the MARCS/Turbospectrum grid (see the text).

the cool $\left(T_{\text {eff }}<4000 \mathrm{~K}\right)$ and C-rich MARCS models atmospheres and we plan to improve the latter C-rich models with new opacities for such polyatomic molecules.

The authors acknowledge the Texas Advanced Computing Center (TACC) at The University of Texas at Austin for providing HPC resources that have contributed to the research results reported within this paper (http://www.tacc.utexas.edu). This paper made use of the IAC Supercomputing facility HTCondor (http://research.cs.wisc.edu/htcondor). O.Z., D.A.G. H., and A.M. acknowledge support provided by the Spanish Ministry of Economy and Competitiveness under grant AYA2011-27754. R.C. acknowledges support provided by the Spanish Ministry of Economy and Competiviness under grants AYA2010-16717 and AYA2013-42781 P. Funding for SDSSIII has been provided by the Alfred P. Sloan Foundation, the Participating Institutions, the National Science Foundation, and the U.S. Department of Energy Office of Science. The SDSS-III web site is http://www.sdss3.org/. SDSS-III is managed by the Astrophysical Research Consortium for the Participating
Institutions of the SDSS-III Collaboration including the University of Arizona, the Brazilian Participation Group, Brookhaven National Laboratory, University of Cambridge, Carnegie Mellon University, University of Florida, the French Participation Group, the German Participation Group, Harvard University, the Instituto de Astrofísica de Canarias, the Michigan State/Notre Dame/JINA Participation Group, Johns Hopkins University, Lawrence Berkeley National Laboratory, Max Planck Institute for Astrophysics, New Mexico State University, New York University, Ohio State University, Pennsylvania State University, University of Portsmouth, Princeton University, the Spanish Participation Group, University of Tokyo, University of Utah, Vanderbilt University, University of Virginia, University of Washington, and Yale University.

\section{REFERENCES}

Ahn, C. P., Alexandroff, R., Allende Prieto, C., et al. 2014, ApJS, 211, 17 Alam, S., Albareti, F. D., Allende Prieto, C., et al. 2015, ApJS, in press (arXiv:1501.00963) 
Allende Prieto, C., Barklem, P. S., Asplund, M., \& Ruiz Cobo, B. 2001, ApJ, 558,830

Allende Prieto, C. 2008, PhST, 133, 014014

Allende Prieto, C., Majewski, S. R., Schiavon, R., et al. 2008, AN, 329, 1018

Alvarez, R., \& Plez, B. 1998, A\&A, 330, 1109

Anstee, S. D., \& O'Mara, B. J. 1995, MNRAS, 276, 859

Asplund, M., Grevesse, N., \& Sauval, A. J. 2005, in ASP Conf. Ser. 336 Cosmic Abundances as Records of Stellar Evolution and Nucleosynthesis in honor of David L. Lambert, ed. T. G. Barnes III \& F. N. Bash (San Francisco, CA: ASP), 25

Barklem, P. S., Piskunov, N., \& O’Mara, B. J. 2000, A\&AS, 142, 467

Bautista, M. A. 1997, A\&AS, 122, 167

Bessell, M. S., Castelli, F., \& Plez, B. 1998, A\&A, 333, 231

Castelli, F., \& Kurucz, R. L. 2003, in IAU Symp. 210, New Grids of ATLAS9 Model Atmospheres, ed. N. Piskunov, W. W. Weiss \& D. F. Gray (Cambridge: Cambridge Univ. Press), 20

Cunto, W., Mendoza, C., Ochsenbein, F., \& Zeippen, C. J. 1993, A\&A, 275, L5

Eisenstein, D. J., Weinberg, D. H., Agol, E., et al. 2011, AJ, 142, 72

García Pérez, A. E., Allende Prieto, C., Holtzman, J. A., et al. 2015, AJ, submitted

González Hernández, J. I., \& Bonifacio, P. 2009, A\&A, 497, 497

Grevesse, N., \& Sauval, A. J. 1998, SSRv, 85, 161

Gunn, J. E., Siegmund, W. A., Mannery, E. J., et al. 2006, AJ, 131, 2332

Gustafsson, B., Edvardsson, B., Eriksson, K., et al. 2008, A\&A, 486, 951

Hall, D. N. B., Ridgway, S., Bell, E. A., \& Yarborough, J. M. 1979, Proc. SPIE, 172, 121

Hauschildt, P. H., Allard, F., Ferguson, J., Baron, E., \& Alexander, D. R. 1999 ApJ, 525, 871

Henyey, L., Vardya, M. S., \& Bodenheimer, P. 1965, ApJ, 142, 841

Heiter, U., \& Eriksson, K. 2006, A\&A, 452, 1039
Hinkle, K., Wallace, L., \& Livingston, W. 1995, PASP, 107, 1042

Holtzman, J. A., Shetrone, M., Johnson, J. A., et al. 2015, AJ, submitted (arXiv:1501.04110)

Hubeny, I., \& Lanz, T. 1995, ApJ, 439, 875

Hubeny, I. 2006, in Computational Methods in Transport, Vol. 794, ed. F. Graziani (Berlin: Springer), 15

Koesterke, L., Allende Prieto, C., \& Lambert, D. L. 2008, ApJ, 680, 764

Koesterke, L. 2009, in AIP Conf. Ser. 1171, Recent Directions in Astrophysical Quantitative Spectroscopy and Radiation Hydrodynamics, ed. I. Hubeny, J. M. Stone, K. MacGregor, \& K. Werner (New York: AIP), 73

Kurucz, R. L. 1993, Kurucz CD-ROM (Cambridge, MA: Smithsonian Astrophysical Observatory)

Kurucz, R. L. 2005, MSAIS, 8, 14

Livingston, W., \& Wallace, L. 1991, National Solar Observatory: An Atlas of the Solar Spectrum in the Infrared from 1850 to $9000 \mathrm{~cm}^{-1}$, Tech. Rep. \#9100 (Tucson, AZ: National Solar Observatory)

Majewski, S. R., Schiavon, R. P., Allende Prieto, C., et al. 2015, AJ, submitted Mészáros, Sz., Allende Prieto, C., Edvardsson, B., et al. 2012, AJ, 144,120

Mészáros, Sz., Holtzman, J., García Pérez, A. E., et al. 2013, AJ, 146, 133

Nahar, S. N. 1995, A\&A, 293, 597

Nidever, D. L., Holtzman, J. A., Allende Prieto, C., et al. 2015, AJ, submitted (arXiv:1501.03742)

Pearson, K. 1901, PMag, 2, 559

Plez, B. 2012, Astrophysics Source Code Library, record ascl:1205.004

Shetrone, M., Bizyaev, D., Lawler, J. E., et al. 2015, ApJS, submitted (arXiv:1502.04080)

Smith, V. V., Cunha, K., Shetrone, M. D., et al. 2013, ApJ, 765, 16

Sneden, C. 1973, ApJ, 184, 839

Wilson, J. C., Hearty, F., Skrutskie, M. F., et al. 2010, Proc, SPIE, 7735, 77351C 